\title{
Flow separation around a square cylinder at low to moderate Reynolds numbers
}

\author{
HONGYI JIANG ${ }^{1}$ (蒋弘毅), and LIANG CHENG ${ }^{1,2 \dagger}$ (程亮) \\ ${ }^{1}$ School of Engineering, The University of Western Australia, 35 Stirling Highway, \\ Perth, WA 6009, Australia \\ ${ }^{2}$ State Key Laboratory of Coastal and Offshore Engineering, Dalian University of \\ Technology, Dalian, 116024, China
}

\begin{abstract}
Flow (of a Newtonian and incompressible fluid) separation around a square cylinder for Reynolds numbers $(R e)$ in the range of 10 to 400 is investigated through direct numerical simulations. In contrast to the general belief that for a square cylinder the flow would always separate at the leading and/or trailing edges of the cylinder, this study shows that the flow (both time-averaged and instantaneous) may not separate at the sharp corners for a certain range of moderate $R e$ values. Instead, separation emerges at approximately a quarter of cylinder length downstream of the leading edge at critical $R e$ values of 100.36 and 96 for the time-averaged and instantaneous flows, respectively. With the increase in $R e$, the time-averaged separation location gradually moves towards the leading edge, while its instantaneous variation range reduces. The evolution of the separation pattern with $R e$ is categorised with a fine $R e$ resolution of 1 . A critical point is identified at $R e=156$ (for the time-averaged flow), where a saddle point emerges away from the upper/lower surface of the cylinder to give rise to wake flow entrainment to the upper/lower side of the cylinder. The rate of the entrained flow is governed by the location of the saddle point. The flow three-dimensionality occurring at $R e>165.7$ affects the location of the saddle point but has almost no influence on the location of the separation point. The corresponding physical mechanism is explained.
\end{abstract}

${ }^{\dagger}$ Correspondence author: liang.cheng@uwa.edu.au 


\section{Introduction}

Bluff-body flow has been a classical topic in fluid mechanics. An important characteristic of bluff-body flow is the flow separation from the body, where the location of separation may affect strongly the hydrodynamic characteristics of the body. The most typical and commonly-used bluff bodies include (i) a circular cylinder and (ii) a square cylinder aligned with sides perpendicular and parallel to the incoming flow (simply referred to as a "square cylinder" hereafter). The scenario of steady incoming flow past a nominally two-dimensional (2D) smooth circular/square cylinder (referred to as "flow past a circular/square cylinder" hereafter) is governed by a single dimensionless parameter, i.e. the Reynolds number $\operatorname{Re}(=U D / v)$, which is defined based on the incoming flow velocity $(U)$, the length scale of the cylinder perpendicular to the incoming flow $(D)$ and the kinematic viscosity of the fluid $(v)$. Specifically, the length scales for a circular and a square cylinder are the diameter and the side length of the cylinder, respectively.

\section{A. Flow separation around a circular cylinder}

Among different bluff bodies, flow separation around a circular cylinder has been studied most extensively in the literature. For a circular cylinder, the flow initially separates at the rear stagnation point of the cylinder (Sen et al., 2009). The critical Re for the onset of flow separation was calculated numerically at $6-7$ in $\mathrm{Wu}$ et al. (2004) and 6.29 in Sen et al. (2009). Based on a cross-validation of the results calculated from 2D numerical simulations and on 2D soap-film experiments, Wu et al. (2004) proposed an empirical formula for the $\bar{\theta}_{s}-R e$ relationship in the 2D (laminar) flow regime:

$\overline{\theta_{s}}=95.7+267.1 R e^{-1 / 2}-625.9 R e^{-1}+1046.6 R e^{-3 / 2} \quad(7 \leq R e \leq 200)$,

where $\theta_{s}$ is the separation angle measured from the front stagnation point of the cylinder to the separation point, and $\overline{\theta_{s}}$ denotes the time-averaged separation angle. 
As indicated by equation (1), in the $2 \mathrm{D}$ flow regime $\bar{\theta}_{s}$ decreases monotonically with increase in $R e$.

As the flow transitions to early three-dimensional (3D) states, specifically from the onset of flow three-dimensionality at $R e \sim 190$ (Williamson, 1996; Barkley and Henderson, 1996; Posdziech and Grundmann, 2001) to $R e \sim 270$, the $\overline{\theta_{s}}$ value is influenced by the consecutive appearance of a few different 3D wake patterns, such that the $\overline{\theta_{s}}-R e$ relationship is no longer monotonic (Jiang, 2020). Beyond the wake transition regimes, the increasingly disordered mode B structure becomes the sole wake pattern for $R e$ from approximately 270 up to at least 10000 (Williamson, 1996), and the 3D $\overline{\theta_{s}}-\operatorname{Re}$ relationship becomes monotonic again (Jiang, 2020):

$\overline{\theta_{s}}=78.8+505 \operatorname{Re}^{-1 / 2} \quad\left(270 \leq \operatorname{Re} \lesssim 10^{5}\right)$.

At $R e \sim 2 \times 10^{5}$ the flow transitions to the critical regime, and the $\overline{\theta_{s}}$ value undergoes a drastic increase from $\sim 80^{\circ}$ to $\sim 140^{\circ}$ (Achenbach, 1968), leading to the so-called "drag crisis" (Wieselsberger, 1922).

\section{B. Flow separation around a square cylinder}

The existing knowledge on flow separation around a square cylinder is summarised below and is compared with that of a circular cylinder summarised in section I.A.

For the case of a square cylinder, the flow initially separates at the middle of the rear surface of the cylinder with a critical Re of 1.15 (Sen et al., 2011). Subsequently, the separation points move towards the two trailing edges of the cylinder over $R e=$ $1.15-5$ and remain at the two trailing edges for $R e \gtrsim 5$, and up to the largest $R e$ of 40 considered in Sen et al. (2011). Different from the case of a circular cylinder, the separation from a square cylinder is in favour of the sharp corners. Based on the 2D numerical studies by Sohankar et al. (1997), Robichaux et al. (1999) and Sharma and Eswaran (2004), with the further increase in $R e$ a few separation patterns have been 
discovered:

(i) Pattern 1 - The flow separates at the two trailing edges only.

(ii) Pattern 2 - The flow separates at not only the two trailing edges but also the two leading edges.

(iii) Pattern 3 - The flow separates at the two leading edges only.

Robichaux et al. (1999) examined the time-averaged flows and reported a transition from pattern 1 to pattern 2 at $R e \sim 120$, followed by a transition from pattern 2 to pattern 3 at $R e \sim 150$. Similarly, Sharma and Eswaran (2004) reported the two transitions for the time-averaged flows at $R e=100-110$ and $150-160$, respectively. On the other hand, Sohankar et al. (1997) examined the instantaneous flows and reported a transition from pattern 1 to pattern 2 at $R e \sim 125$, followed by a transition from pattern 2 to pattern 3 at $R e \sim 175$. Pattern 3 remains as the separation pattern for the 3D flows at higher $R e$ values, including $R e=200-500$ investigated in Sohankar et al. (1999), $R e=550-3400$ investigated in Ozgoren (2006), and $R e$ up to $4.5 \times 10^{4}$ in Bai and Alam (2018).

In summary, in contrast to the case of a circular cylinder where the time-averaged separation point generally moves upstream with increase in Re before the boundary layer transitions to turbulence at $\operatorname{Re} \sim 2 \times 10^{5}$, it is generally believed that for a square cylinder the flow (both instantaneous and time-averaged) would always separate at the leading and/or trailing edges owing to the geometric sharp corners.

Nevertheless, it is noticed from some recent studies that for some other sharp-cornered bluff bodies the flow may not always separate at the sharp corners. For example, for an inclined triangular cylinder investigated in $\mathrm{Ng}$ et al. (2016) (Fig. 1a) and an inclined square cylinder investigated in Kumar et al. (2018) (Fig. 1b) the steady flow first separates on the cylinder surfaces rather than at one of the sharp corners. In addition, Yoon et al. (2010) examined a series of inclined square cylinders with various flow incidence angles $(\theta)$ to the upper and lower surfaces of the cylinder ranging from $\theta=0^{\circ}$ (i.e. a square cylinder) to $\theta=45^{\circ}$ (i.e. a diamond cylinder) at a fixed $R e$ of 60 (see e.g. an inclined square cylinder with $\theta=10.2^{\circ}$ in Fig. 1c). While the separation points determined from the time-averaged flow locate at edges $\mathrm{C}$ and $\mathrm{D}$ 
for $\theta=0^{\circ}$ and edges $\mathrm{B}$ and $\mathrm{D}$ for $\theta=45^{\circ}$, for a certain range of $\theta$ between $0-45^{\circ}$ the time-averaged upper separation point moves gradually from edge $\mathrm{C}$ to edge $\mathrm{B}$ (see e.g. an example shown in Fig. 1c) (Yoon et al., 2010). For the case of $\theta=10.2^{\circ}$ shown in Fig. 1(c), Yoon et al. (2010) also examined the instantaneous flow fields over a vortex shedding cycle and found that the instantaneous upper separation point moves back and forth on the surface BC.

(a)

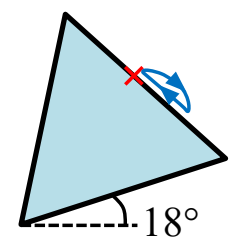

(b)

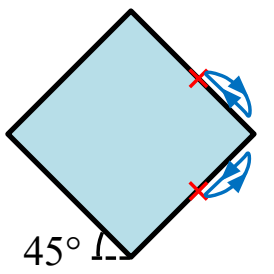

(c)

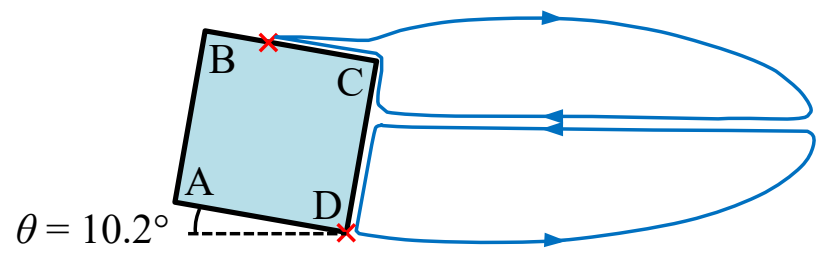

Fig. 1. Sketch of the flow separation pattern for (a) an inclined triangular cylinder at the initiation of separation of $R e \sim 7$ (Ng et al., 2016), (b) an inclined square cylinder at the initiation of separation of $R e \sim 7.3$ (Kumar et al., 2018), and (c) an inclined square cylinder at $R e=60$ (Yoon et al., 2010). The recirculation bubbles are shown in blue while the separation points are marked by red crosses.

In consideration of the possibility that the flow may not always separate at the sharp corners of the cylinder, this study will re-visit and refine (using a refined $R e$ interval of 1) the 2D separation patterns of a square cylinder reported earlier in Sohankar et al. (1997), Robichaux et al. (1999) and Sharma and Eswaran (2004). It will be shown in this study that for a square cylinder the flow may not separate at the sharp corners for a certain range of moderate $R e$ values.

In addition, the separation patterns for a square cylinder will be examined for $R e$ up to the $3 \mathrm{D}$ wake transition regimes ranging from the onset of three-dimensionality at $R e \sim 166$ (e.g. Sheard et al., 2009; Choi et al., 2012; Park and Yang, 2016) to Re= 
400 , revealing the effects of three-dimensionality on flow separation.

The remainder of this paper is organised as follows. The numerical method and computational domain/mesh are presented in section II. Evidence for the non-edge separation around a square cylinder is demonstrated in section III.A, followed by the investigation of time-averaged and instantaneous separation patterns in sections III.B and III.C, respectively. Finally, major conclusions are drawn in section IV.

\section{Numerical model}

\section{A. Numerical method}

Direct numerical simulations (DNS) have been performed in this study based on the framework of an open-source code OpenFOAM (www.openfoam.org). The governing equations for the flow are the continuity and incompressible Navier-Stokes equations:

$\frac{\partial u_{i}}{\partial x_{i}}=0$

$\frac{\partial u_{i}}{\partial t}+u_{j} \frac{\partial u_{i}}{\partial x_{j}}=-\frac{1}{\rho} \frac{\partial p}{\partial x_{i}}+v \frac{\partial^{2} u_{i}}{\partial x_{j} \partial x_{j}}$

where $\left(x_{1}, x_{2}, x_{3}\right)=(x, y, z)$ are Cartesian coordinates, $u_{i}$ is the velocity component in the direction $x_{i}, t$ is time, $\rho$ is fluid density, $p$ is pressure, and $v$ is kinematic viscosity. The advection, diffusion and time derivative terms are discretized, respectively, using a fourth-order cubic scheme, a second-order linear scheme, and a blended scheme consisting of the second-order Crank-Nicolson scheme and a first-order Euler implicit scheme. The same numerical approach has been adopted in Jiang et al. $(2016,2018)$ for the simulations of wake transition of a circular and a square cylinder. More details on this numerical approach are referred to in Jiang and Cheng (2018).

\section{B. Computational domain and mesh}

As sketched in Fig. 2(a), a hexahedral computational domain is adopted for the 
simulation of flow past a square cylinder. The computational domain size is $L_{x} \times L_{y} \times L_{z}$ $=(30 D+30 D) \times(30 D+30 D) \times 15 D$, with $L_{x}, L_{y}$, and $L_{z}$ being the domain sizes in the $x$-, $y$-, and $z$-directions, respectively. A square cylinder is placed at the centre of the $x-y$ plane. The boundary conditions are listed in Table 1 , where $\partial / \partial n=0$ denotes a zero normal gradient condition. The incoming flow is uniform and is free of turbulence disturbance. The internal flow follows an impulsive start, i.e. at the beginning of the simulation the velocity and pressure of each cell in the computational domain are set to zero.

(a)

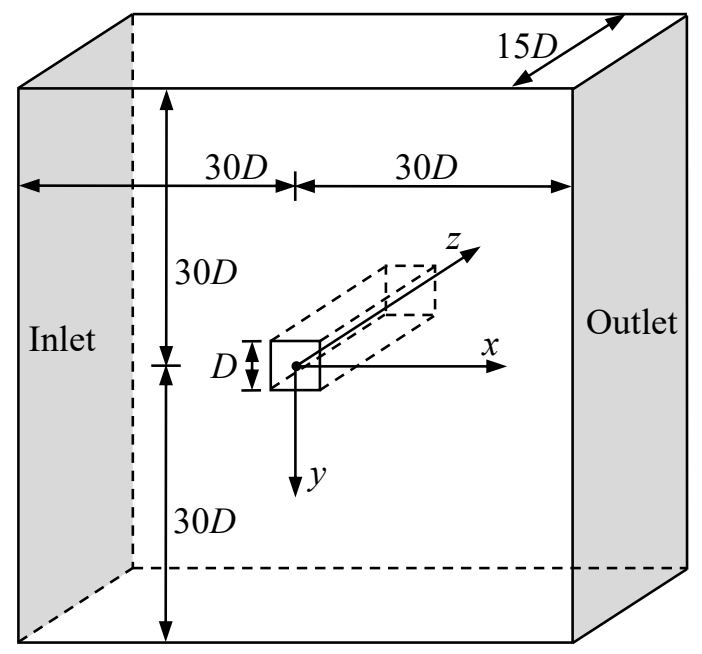

(b)

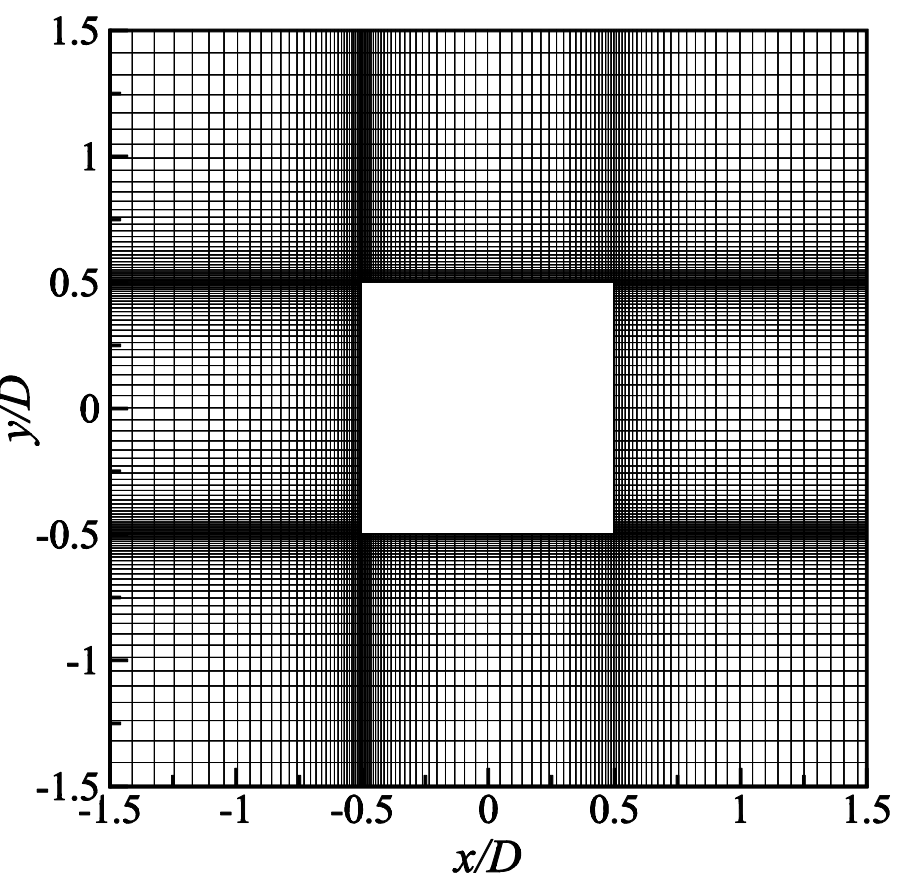

Fig. 2. The computational domain and mesh: (a) A schematic model of the 
computational domain (not to scale), and (b) A close-up view of the 2D reference mesh (with $F_{m r}=1$ ) near the cylinder. The mesh selected for the present study (with $\left.F_{m r}=4\right)$ can be viewed as a mesh with only each fourth grid line turned on in panel (b).

Table 1. Boundary conditions for the computational domain.

\begin{tabular}{llll}
\hline Boundary & Type & Velocity components & Pressure \\
\hline Inlet & Inflow & $u_{x}=U, u_{y}=u_{z}=0$ & $\partial p / \partial n=0$ \\
Outlet & Outflow & $\partial u_{i} / \partial n=0$ & $p=0$ \\
Top and bottom & Symmetry & $\partial u_{x} / \partial n=\partial u_{z} / \partial n=0, u_{y}=0$ & $\partial p / \partial n=0$ \\
Front and back & Periodic & $u_{i}(x, y, z=0, t)=u_{i}\left(x, y, z=L_{z}, t\right)$, & $p(x, y, z=0, t)=p\left(x, y, z=L_{z}, t\right)$, \\
& & $\partial u_{i} / \partial z(x, y, z=0, t)=\partial u_{i} / \partial z(x, y$, & $\partial p / \partial z(x, y, z=0, t)=\partial p / \partial z(x, y, z$ \\
& & $\left.z=L_{z}, t\right)$ & $\left.=L_{z}, t\right)$ \\
Cylinder surface & No-slip & $u_{i}=0$ & $\partial p / \partial n=0$ \\
\hline
\end{tabular}

The computational mesh for the present study is a modification of the "reference mesh" used in Jiang et al. (2018) for the simulations of 3D wake transition of a square cylinder for $R e$ up to 400 . The reference mesh is introduced in this section and specific modifications are detailed in section III.A.

Some key parameters of the reference mesh are given below. For the 2D mesh in the $x-y$ plane, the number of cells around the cylinder surface is 196 . The smallest cell size at the leading edges of the cylinder (where largest pressure gradients take place) is $0.005 D \times 0.005 D$. The cell expansion ratio in the whole domain is kept below 1.1. To capture detailed wake structures, a relatively high mesh resolution is used in the near wake by specifying the streamwise mesh size varying linearly from $0.05 D$ at $x / D=1$ to $0.1 D$ at $x / D=7$. A close-up view of the $2 \mathrm{D}$ mesh near the cylinder is shown in Fig. 2(b). The corresponding $3 \mathrm{D}$ mesh is constructed by replicating the $2 \mathrm{D}$ mesh along the $z$-axis with a spanwise cell size of $0.125 D$, which results in an identical mesh resolution in all 120 planes perpendicular to the spanwise direction. 
A 3D mesh convergence study has been reported separately in Jiang et al. (2018) at $R e=400$ (the largest $R e$ adopted in the present study) with two variations to the above-mentioned reference mesh, one with the numbers of cells in both the $x$ - and $y$-directions 1.5 times that of the reference mesh (and the total number of cells 2.25 times that of the reference mesh), and the other with a reduced spanwise cell size from $0.125 D$ to $0.0625 D$ (and the total number of cells twice that of the reference mesh). It has been shown in Jiang et al. (2018) that the hydrodynamic forces on the cylinder and the velocity profiles in the near wake calculated with the two refined meshes are very close to those calculated with the reference mesh. Specifically, the relative differences are within $0.5 \%$ for the Strouhal number and time-averaged drag coefficient, and within $3.5 \%$ for the root-mean-square lift coefficient.

The time step size $(\Delta t)$ for each case is chosen based on a Courant-FriedrichsLewy (CFL) limit of 0.5 , where the CFL number is defined as

$\mathrm{CFL}=\frac{|u| \Delta t}{\Delta l}$,

where $|u|$ is the magnitude of the velocity through a cell, and $\Delta l$ is the cell size in the direction of the velocity. As an example, by using the reference mesh the time step size for $R e=400$ is $1.47 \times 10^{-3}$.

\section{Numerical results}

\section{A. Evidence for the non-edge separation}

For flow past a square cylinder, the separation and reattachment points on the upper and lower surfaces of the cylinder are determined quantitatively based on the streamwise velocity profiles sampled at the cell centres of the first layer of mesh next to the cylinder surfaces. The separation and reattachment points are identified at the streamwise locations where the streamwise velocity changes its sign, since flow separation and reattachment are associated with flow reversal.

An example for the determination of the time-averaged separation and reattachment locations at $R e=110$ (based on the reference mesh) is shown in Fig. 3. 
Two separation/reattachment points are identified in Fig. 3(a) at the locations where the time-averaged streamwise velocity profile intersects the horizontal line $\overline{u_{x}} / U=$ 0 (in contrast to the case of $R e=100$ where separation/reattachment points do not exist). The separation/reattachment points identified in Fig. 3(a) are confirmed quantitatively through visualization of the streamlines of the time-averaged flow field near the cylinder surface. For example, Fig. 3(b) highlights the streamlines near the upper left corner of the square cylinder. A recirculation region is observed on the upper surface of the cylinder. A separation point and a reattachment point are identified at the left and right ends of the recirculation region, respectively.

(a)

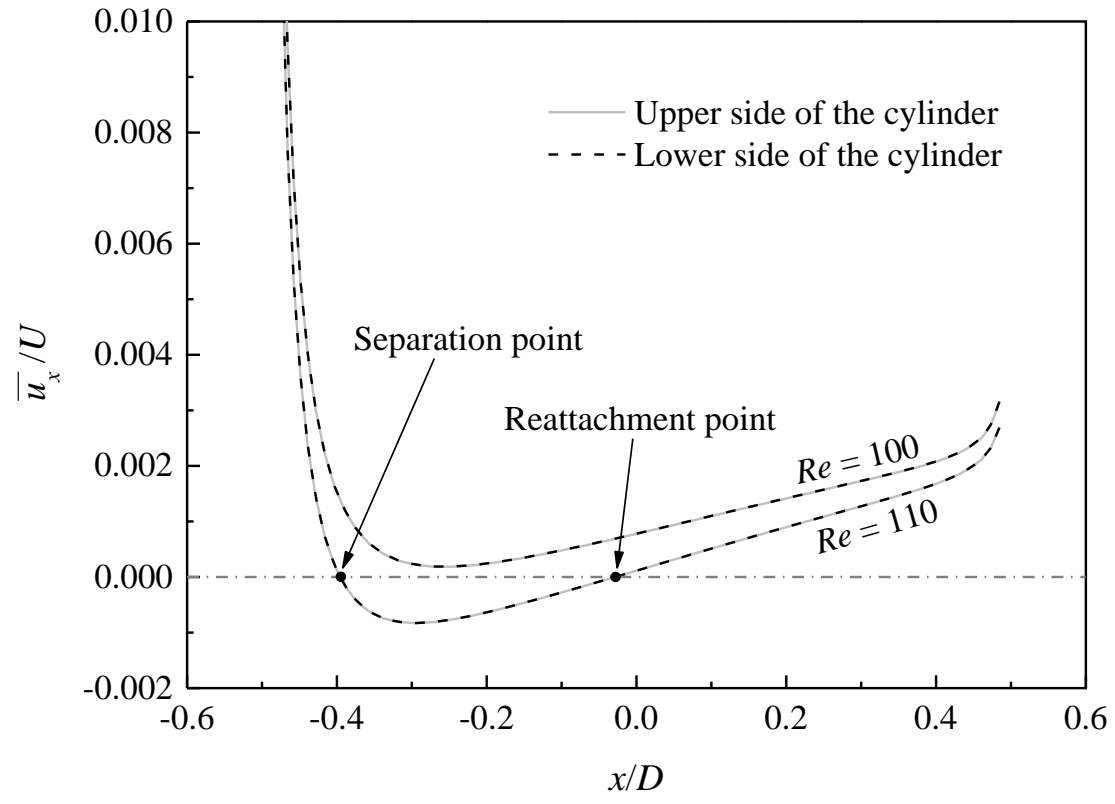

(b)

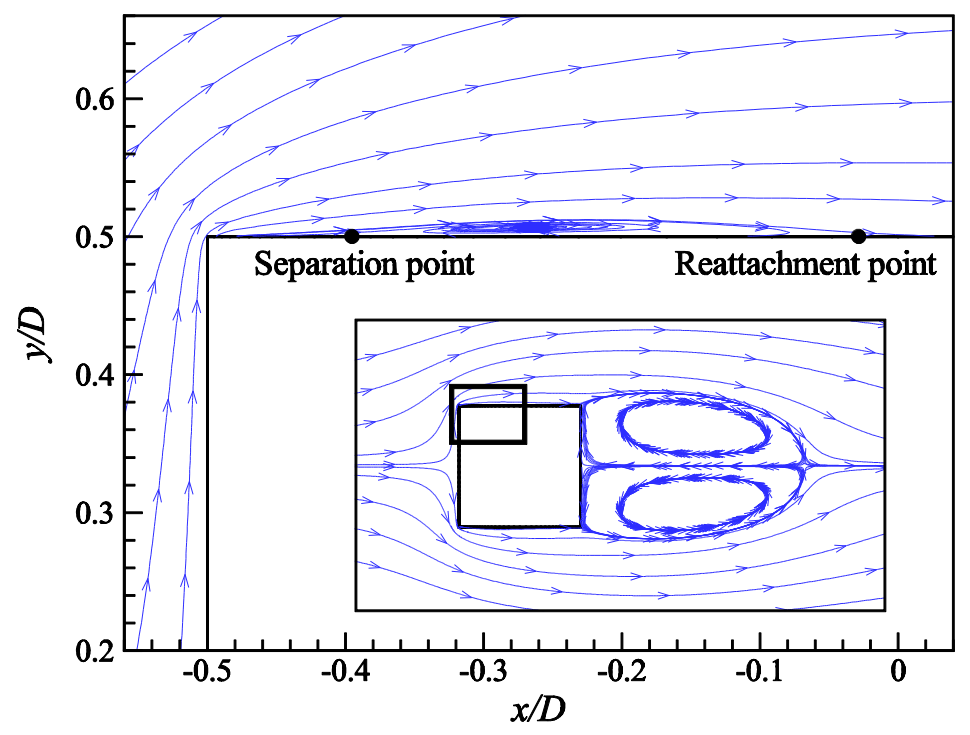


Fig. 3. An example for the determination of the separation and reattachment points. (a) Time-averaged streamwise velocity profiles sampled at the cell centres of the first layer of mesh next to the upper and lower surfaces of the cylinder, and (b) streamlines of the time-averaged flow field for $R e=110$. The streamlines are shown at near the upper left corner of the square cylinder, as marked by a rectangular frame in the inset.

In contrast to the general belief introduced in section I that for flow past a square cylinder the flow would always separate at the leading and/or trailing edges of the cylinder, the counter example shown in Fig. 3 suggests that the flow may not separate at the edge at $R e=110$. This is a new finding, which warrants some further investigation to ensure that it is not induced by numerical issues.

In addition to the mesh convergence study on the prediction of the hydrodynamic forces and velocity profiles mentioned in section II.B, an additional mesh convergence study on the prediction of the separation and reattachment points is performed herein. The mesh resolution in the $x-y$ plane is examined through a few refinements of the reference mesh introduced in section II.B, while the general topology of the mesh remains unchanged. The resolution of a mesh is quantified by the mesh resolution factor $F_{m r}$ (Jiang et al., 2017):

$F_{m r}=\left(\frac{\text { Total cell number of the current mesh }}{\text { Total cell number of the reference mesh }}\right)^{1 / 2}$.

Naturally, $F_{m r}=1$ refers to the reference mesh. For a mesh with a mesh resolution factor $F_{m r}$, the numbers of cells in both the $x$-and $y$-directions are $F_{m r}$ times that of the reference mesh, and the total number of cells is $F_{m r} \times F_{m r}$ times that of the reference mesh. In particular, the number of cells around the cylinder surface is $F_{m r}$ times that of the reference mesh, while the smallest cell size next to the cylinder surface is approximately $1 / F_{m r}$ times that of the reference mesh. In addition, the time step size is reduced by $F_{m r}$ times so as to satisfy the same CFL limit of 0.5 . The computational cost for the case with a mesh resolution factor $F_{m r}$ is at least $F_{m r}{ }^{3}$ times that of the reference mesh.

To ensure a sufficient precision for the determination of the separation and 
reattachment points, the present mesh convergence study adopts refined meshes up to $F_{m r}=6$, which is much finer than that of $F_{m r}=1.5$ used for the mesh convergence study of the hydrodynamic forces in Jiang et al. (2018) (which results in relative errors in the hydrodynamic forces in the order of $1 \%$ ). Here the mesh convergence study is performed for two cases with $R e=105$ and 125, and Fig. 4 shows the separation and reattachment locations predicted with various $F_{m r}$ values. A curve fitting of the relationship between the separation location and $F_{m r}$ (with the coefficient of determination $\mathrm{R}^{2}$ very close to 1 ) suggests that as $F_{m r} \rightarrow \infty$ the separation locations for $R e=105$ and 125 converge to $x / D=-0.3817$ and -0.4487 , respectively (Fig. 4a,c), instead of the generally believed leading edge at $x / D=-0.5$. These two cases show that the non-edge separation is not induced by an insufficient mesh resolution near the cylinder surface or edge.

(a)

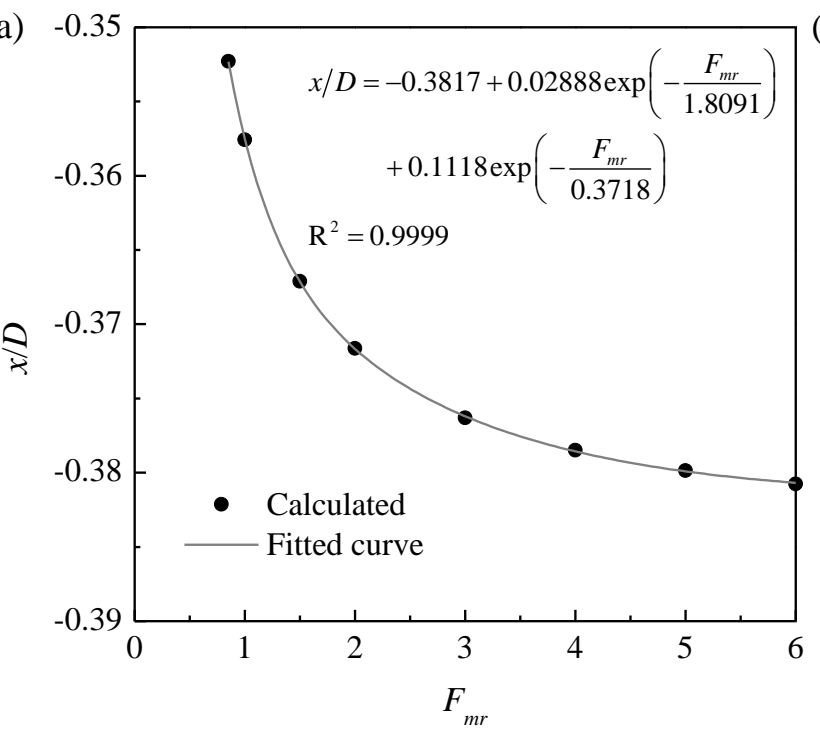

(b)

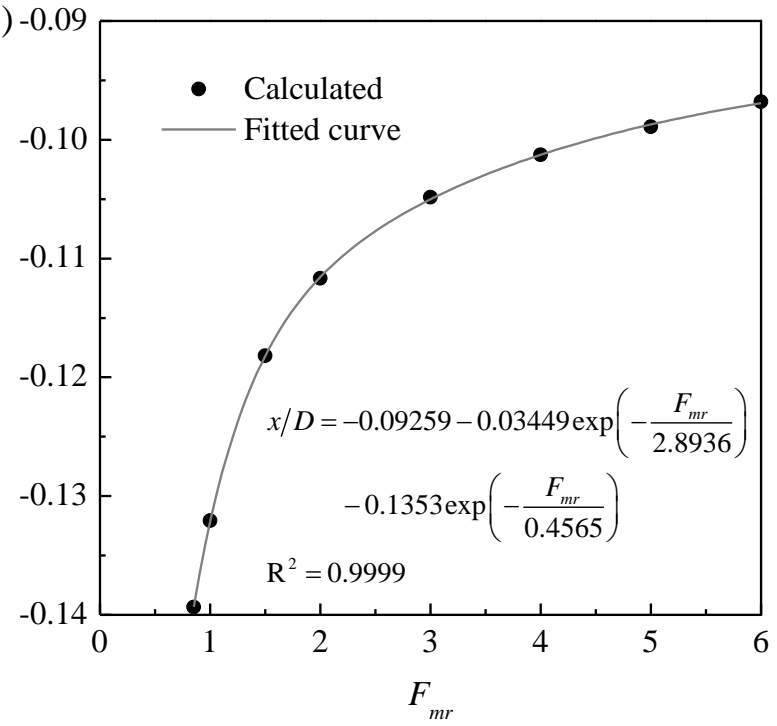



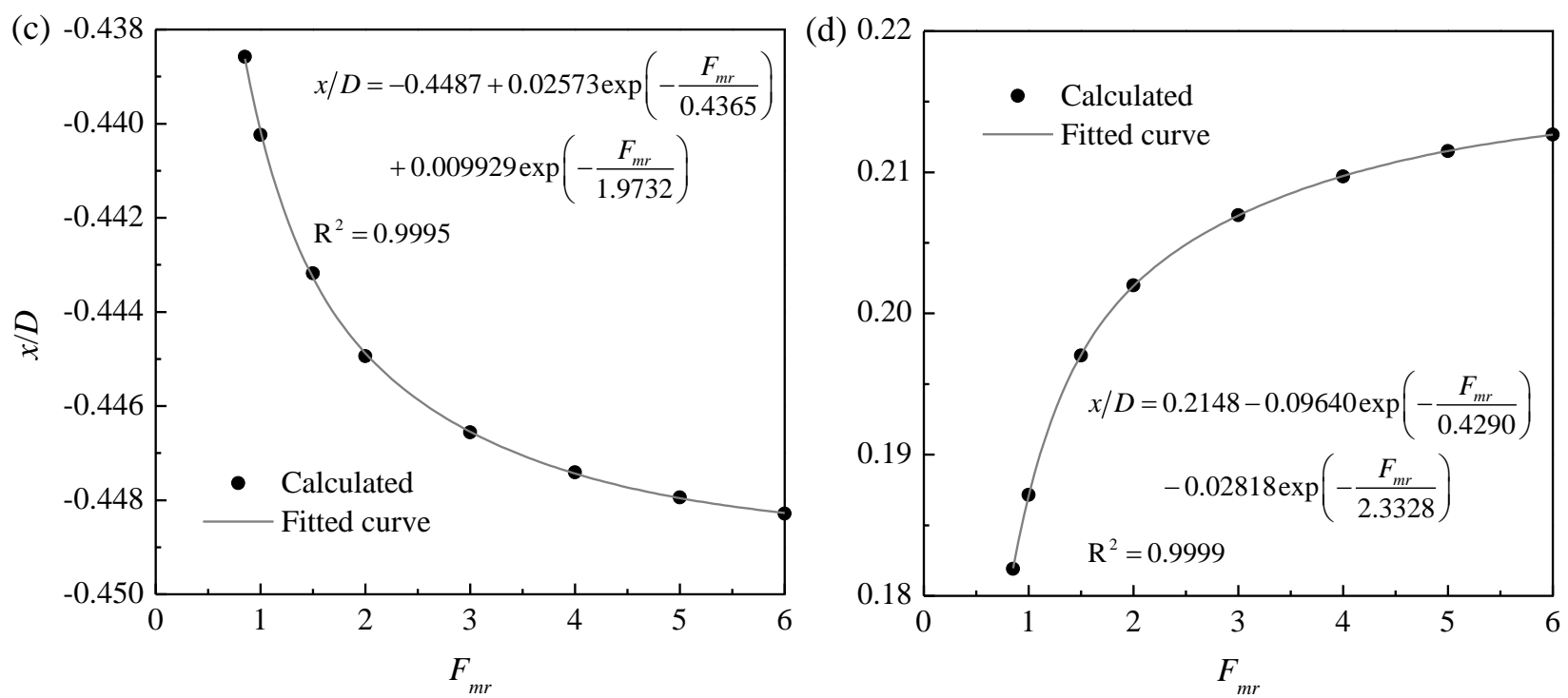

Fig. 4. Mesh convergence study on the prediction of (a) the separation location at $R e$ $=105$, (b) the reattachment location at $R e=105$, (c) the separation location at $R e=$ 125 , and (d) the reattachment location at $R e=125$.

As a compromise between the computational cost and numerical accuracy, the mesh with $F_{m r}=4$ is adopted for the present study, namely the number of cells around the cylinder surface is 784 , the smallest cell size at the leading edges of the cylinder is $0.00125 D \times 0.00125 D$, and the cell expansion ratio in the whole domain is kept below 1.025. Based on the mesh resolution of $F_{m r}=4$, the adequacy of the computational domain size is examined. Table 2 lists the separation and reattachment locations for $R e=125$ predicted by three cases:

(i) Case 1 adopts the mesh used for the present study (with $F_{m r}=4$ ),

(ii) Case 2 doubles the computational domain size from the default set-up of

$$
L_{x} \times L_{y}=(30 D+30 D) \times(30 D+30 D) \text { to }(60 D+60 D) \times(60 D+60 D) \text {, and }
$$

(iii) Case 3 shows the results with an increase in $F_{m r}$ from 4 to 6 .

As shown in Table 2, the increases in the domain size and mesh resolution have opposite effects on the prediction of the separation and reattachment locations. In particular, the influences from cases 2 and 3 are in similar levels and may largely cancel out. This coincidence suggests that $F_{m r}=4$ may be a better choice than $F_{m r} \rightarrow$ $\infty$ in achieving a higher overall precision. 
Table 2. Mesh convergence study on the prediction of the separation and reattachment locations for $R e=125$. The values in the parentheses are the differences with respect to those predicted by case 1 .

\begin{tabular}{llll}
\hline Case & Case description & Separation location $x / D$ & Reattachment location $x / D$ \\
\hline 1 & Mesh for the present study & -0.4474 & 0.2097 \\
& (with $\left.F_{m r}=4\right)$ & & \\
2 & Doubling the domain size & $-0.4467(+0.0007)$ & $0.2053(-0.0044)$ \\
3 & Increase in $F_{m r}$ from 4 to 6 & $-0.4483(-0.0009)$ & $0.2127(+0.0030)$ \\
\hline
\end{tabular}

To further rule out the influence of the numerical set-up (which is based on the framework of OpenFOAM) on the prediction of the separation location, cross-checks have been carried out by using a different open-source code Nektar++ (Cantwell et al., 2015) that is based on a high-order spectral/hp element method. The embedded unsteady Navier-Stokes solver is selected for the present study, together with a second-order implicit-explicit (IMEX) time-integration scheme, a velocity correction scheme, and a continuous Galerkin projection. The same numerical approach has been used in Jiang and Cheng (2019) for the simulations of flow past a circular cylinder.

For the present case of flow past a square cylinder, the computational domain size used in Nektar++ is the same as that used in OpenFOAM. Key parameters of the macro-element mesh include the use of 120 macro-elements around the cylinder perimeter and $0.02 D \times 0.02 D$ for the smallest macro-element size at the leading edges of the cylinder. The macro-elements are further subdivided using fourth-order Lagrange polynomials $\left(N_{p}=4\right)$ on the Gauss-Lobatto-Legendre points for the quadrilateral expansions. For the case of $R e=125$, the separation and reattachment locations predicted by Nektar++ are $x / D=-0.4482$ and 0.2165 , respectively, matching very well those predicted by OpenFOAM using $F_{m r}=6(x / D=-0.4483$ and 0.2127 , respectively), which suggests that both computational codes yield reliable results. In addition, the convergence of the separation location predicted by Nektar++ is 
demonstrated by an almost identical result of $x / D=-0.4484$ predicted with a reduced $N_{p}$ of 3. In summary, the Nektar++ results confirm the existence of a non-edge separation.

\section{B. Time-averaged separation and reattachment locations}

While Sen et al. (2011) focused on the flow separation in the steady regime of $R e$ from 1.15 up to 40, the present study extends the range of $R e$ from 10 up to 400, covering the onset of the primary wake instability at $R e \sim 45$ (e.g. Saha et al., 2000; Park and Yang, 2016) and the onset of the secondary wake instability at $R e \sim 166$ (e.g. Sheard et al., 2009; Choi et al., 2012; Park and Yang, 2016). In the steady regime of $R e \lesssim 45$, the separation location is determined from the fully developed steady flow field. In the unsteady regime of $R e \gtrsim 45$, the instantaneous separation and reattachment locations are determined from the corresponding instantaneous flow field, while the time-averaged locations are determined from the time-averaged flow field. For the time-periodic 2D flows, the time-averaged flow field is obtained by averaging a fully developed vortex shedding period. For the irregular 3D flows, the time-averaged flow field is obtained by averaging at least 800 non-dimensional time units (defined as $t^{*}=t U / D$ ) of the fully developed flow (after discarding an initial period of at least 1000 non-dimensional time units). The results in the 3D regime have also been averaged over the 120 planes in the spanwise direction.

\section{Two-dimensional flows $(\operatorname{Re}<165.7)$}

The evolution of the time-averaged separation, reattachment and saddle points with $R e$ is investigated first. Figure 5 shows schematically the streamlines of the time-averaged 2D flows and the associated separation, reattachment and saddle points for different ranges of $R e$. Since the time-averaged flow is symmetrical about the wake centreline, the discussion below is based on the upper half of the flow field only. 
(a)

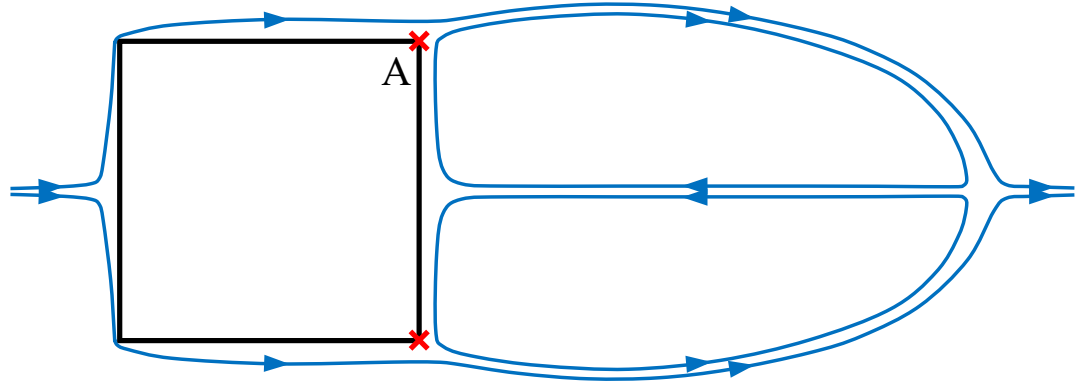

(b)

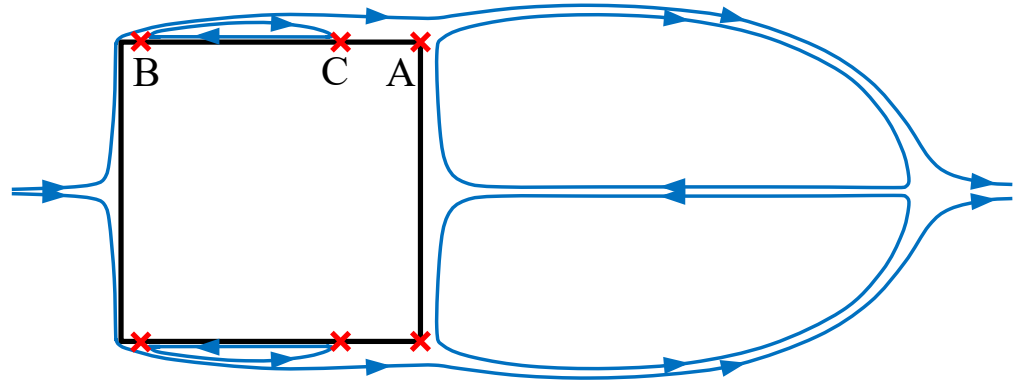

(c)

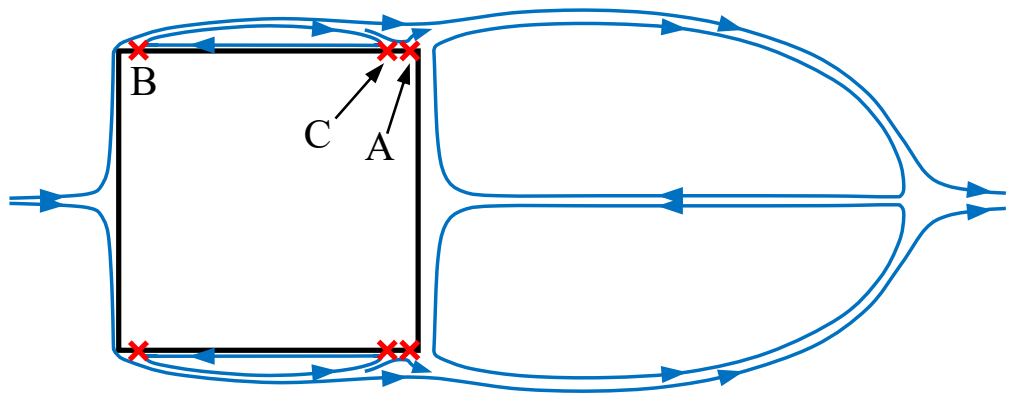

(d)

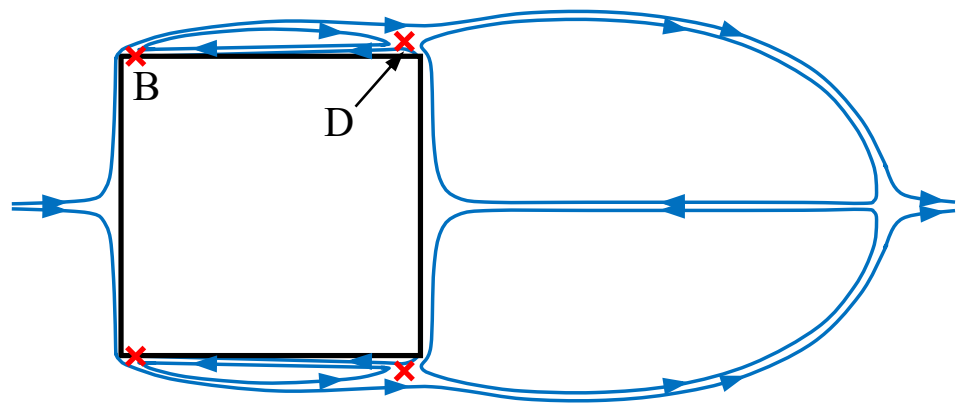

(e)

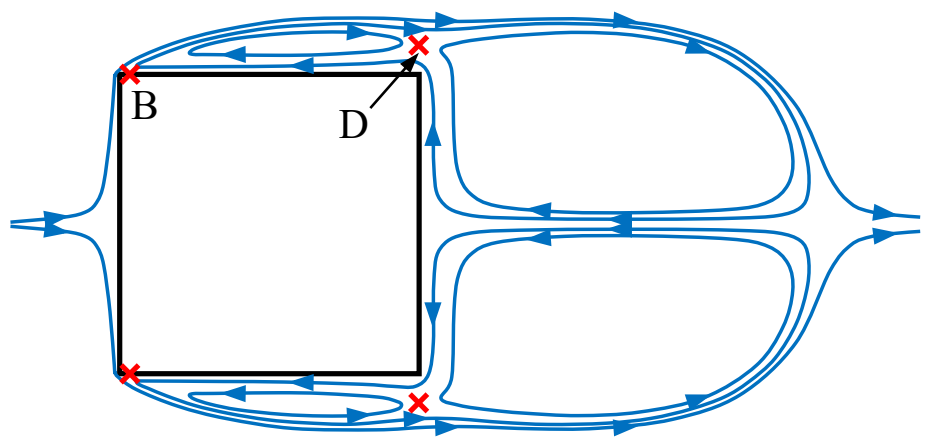

Fig. 5. Schematic diagrams of the streamlines of the time-averaged flow and the associated separation, reattachment and saddle points for (a) $R e=10-100$, (b) $R e=$ 
$101-130$, (c) $R e=131-155$, (d) $R e=156-158$, (e) $R e \geq 159$. The separation, reattachment and saddle points are marked by red crosses. Real flow examples for panels (a), (b), (c) and (e) are shown in Fig. 6(a,b), Fig. 3(b), Fig. 8(a) and Fig. 8(b), respectively.

For $R e=10-100$, the time-averaged flow separates only at the trailing edge of the cylinder, as indicated by point A in Fig. 5(a). In particular, there is no change in the separation pattern when the flow transitions from steady to unsteady at $R e=46$, as evidenced by the similar patterns of streamlines of the time-averaged flows for $R e=$ 45 (Fig. 6a) and $R e=46$ (Fig. 6b). In addition, for $R e=46-100$ not only the time-averaged flow but also the instantaneous flow show that the separation points are fixed at the two trailing edges of the cylinder. As an example, Fig. 6(c,d) shows the instantaneous flows at $R e=46$ of half of a vortex shedding period apart.

(a)

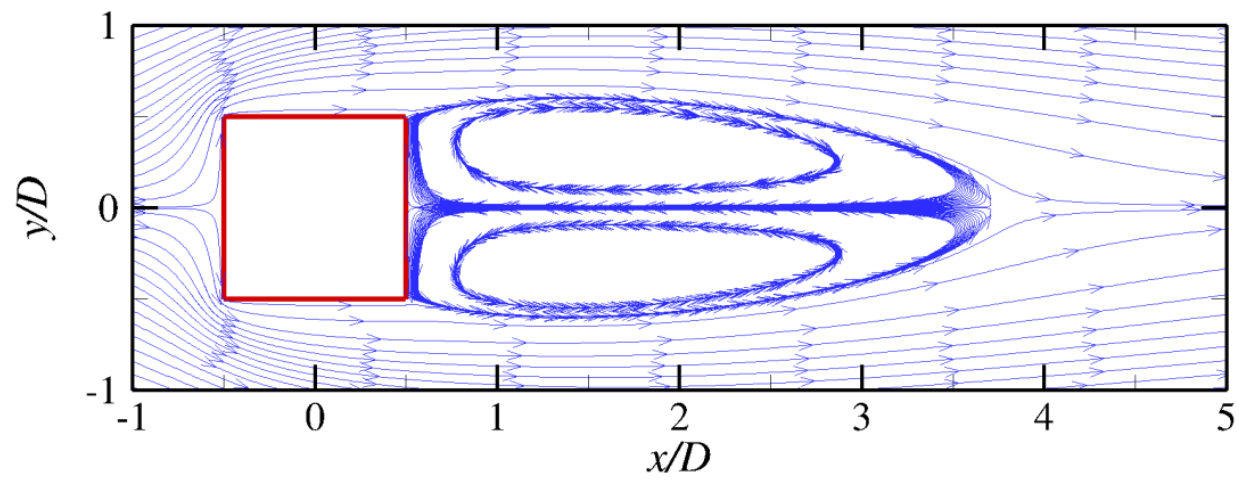

(b)

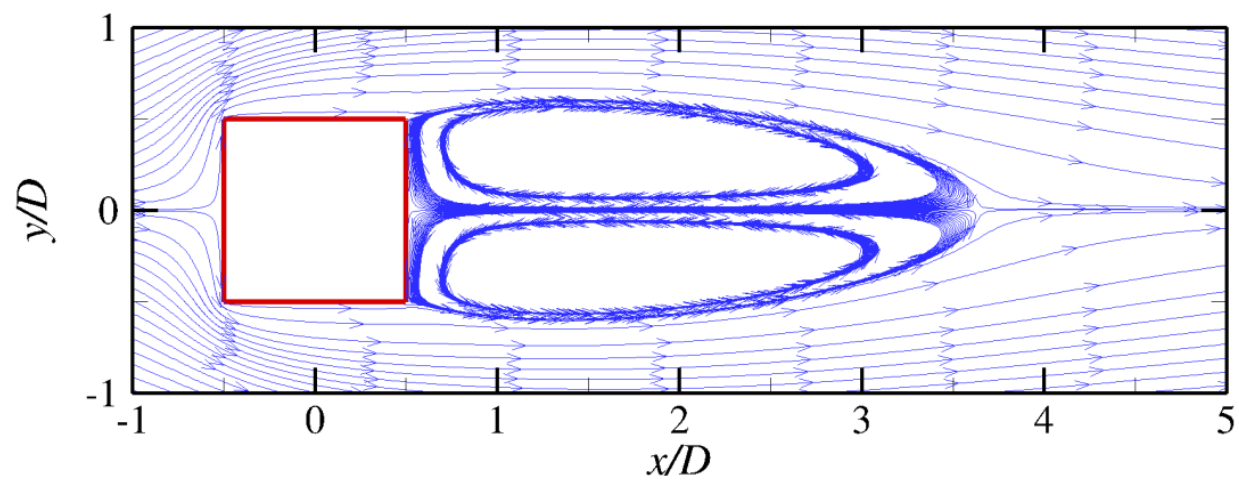


(c)

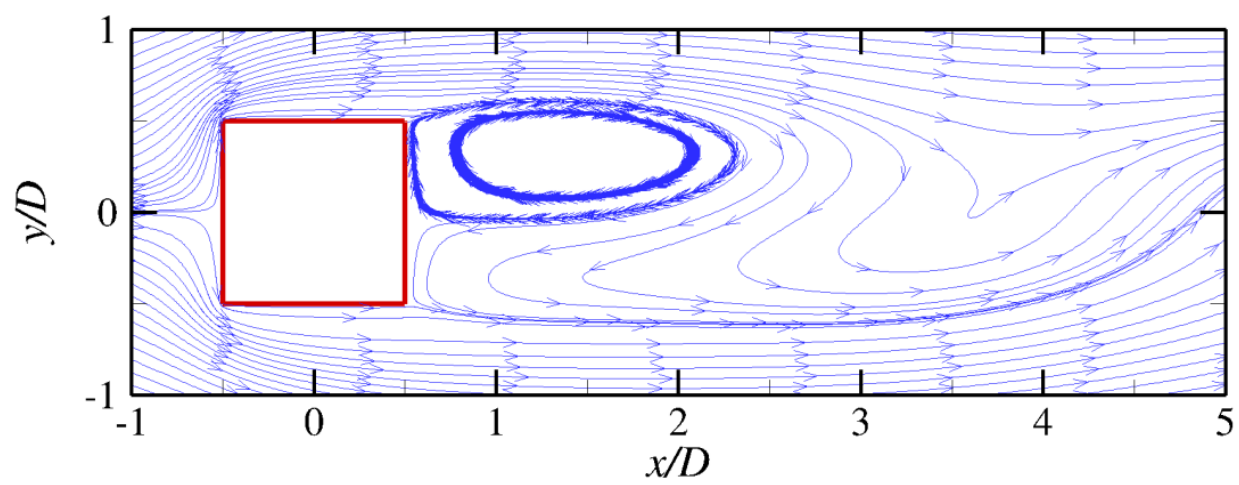

(d)

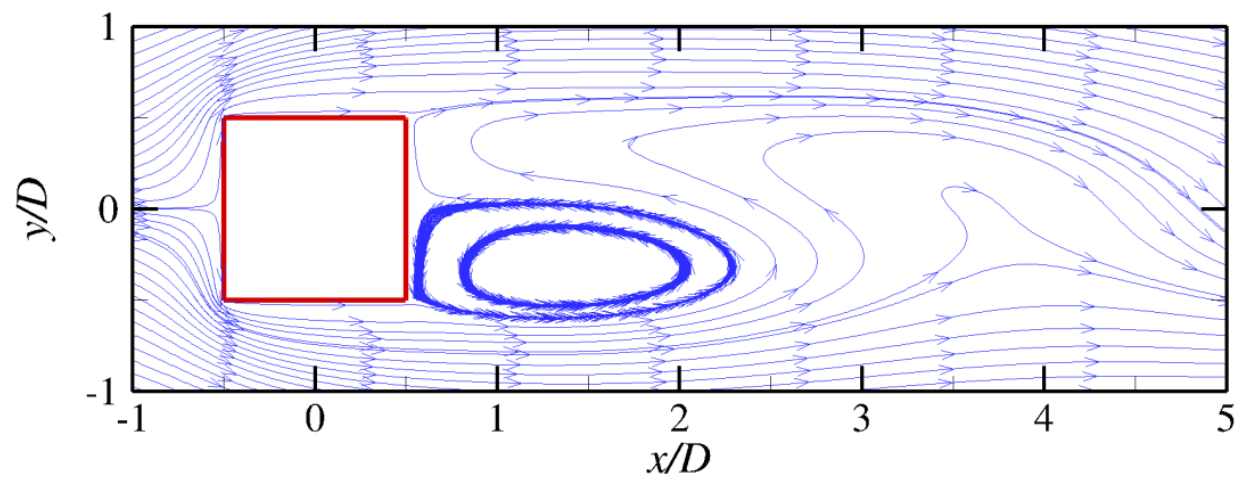

Fig. 6. Streamlines for (a) the time-averaged flow at $R e=45$, (b) the time-averaged flow at $R e=46$, and (c) and (d) the instantaneous flows at $R e=46$ of half of a vortex shedding period apart.

For $R e=101-155$, in addition to the separation point A near the trailing edge, the flow also separates at a short distance downstream of the leading edge (point B in Fig. 5b,c). An example is shown in Fig. 3(b) at $R e=110$. The separation near the leading edge results in the formation of a recirculation region on the upper side of the cylinder and the emergence of a reattachment point $\mathrm{C}$ (Fig. 5b,c). Figure 7(a) quantifies the movements of the time-averaged points $\mathrm{A}, \mathrm{B}$ and $\mathrm{C}$ on the upper surface of the cylinder with $\operatorname{Re}$. At $R e=101$, points $\mathrm{B}$ and $\mathrm{C}$ locate at $x / D=-0.319$ and 0.216 , respectively, namely the upper recirculation region is already $0.103 D$ in length. To further identify the location for the first emergence of this recirculation region, additional simulations are performed within $R e=100-101$, specifically at $R e=$ 100.3 and 100.5. For $R e=100.5$, the time-averaged streamwise velocity profile sampled at the cell centres of the first layer of mesh next to the upper surface of the cylinder intersects $\overline{u_{x}} / U=0$ twice (similar to the case $R e=110$ shown in Fig. 3a), 
while for $R e=100.3$ no such intersection exists (similar to the case $R e=100$ shown in Fig. 3a). Based on an interpolation of the velocity profiles at $R e=100.3$ and 100.5 to a critical condition where only one intersection exists, the critical $R e$ for the emergence of points B and $\mathrm{C}$ is determined at 100.36. At $R e=100.36$, points $\mathrm{B}$ and $\mathrm{C}$ (and a minuscule recirculation region) first emerge at $x / D=-0.271$ (Fig. 7a). With the increase in $R e$ from 100.36 to 155 , point $\mathrm{B}$ moves upstream while point $\mathrm{C}$ moves downstream (Fig. 7a), which indicates a gradual increase in the size of the recirculation region on the upper surface of the cylinder.

(a)

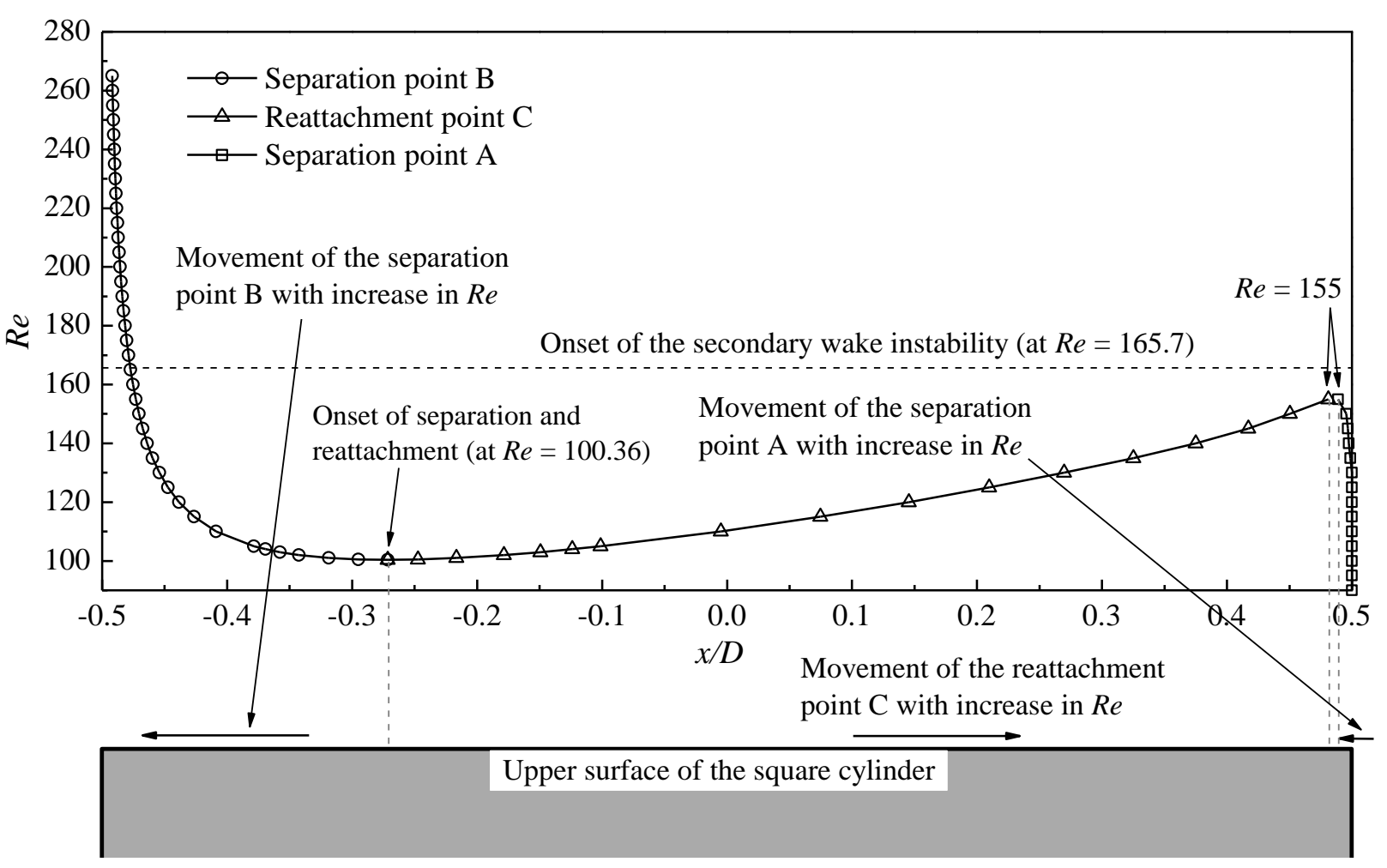


(b)

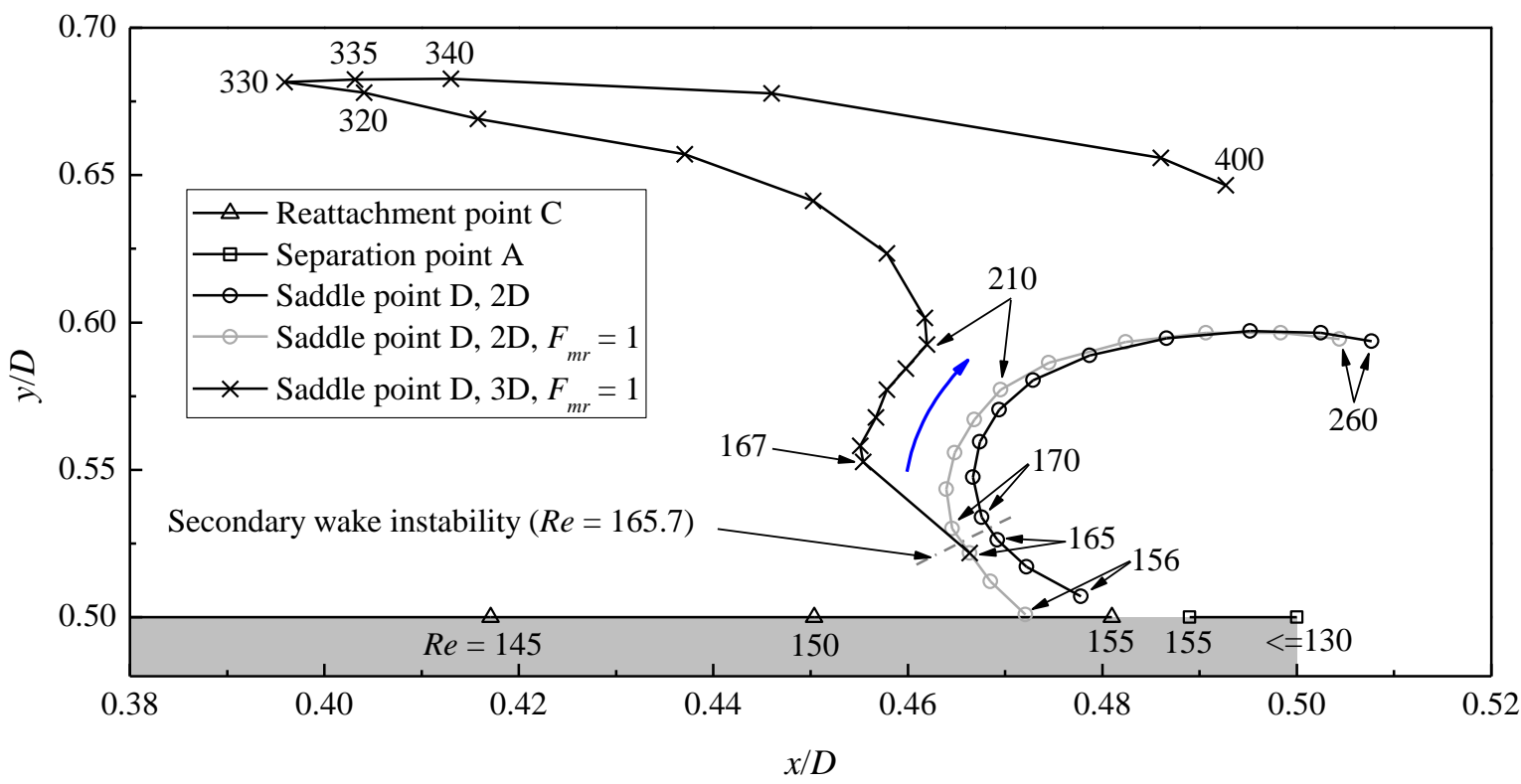

Fig. 7. Movements of the time-averaged separation, reattachment and saddle points with the evolution of Re. (a) The separation and reattachment points A, B and C, and (b) from the concurrent disappearance of the points $\mathrm{A}$ and $\mathrm{C}$ to the emergence of the saddle point $\mathrm{D}$. The shaded area represents the square cylinder.

For $R e=131-155$, while the reattachment point $\mathrm{C}$ approaches the trailing edge, the separation point A also moves slightly upstream (Fig. 5c and Fig. 7a). At $R e=155$, points $\mathrm{A}$ and $\mathrm{C}$ almost meet each other (Fig. 7). For $R e \geq 156$, with the further increase in the size of the recirculation region, the flow separated from point B no longer reattaches to the upper surface of the cylinder. Points $\mathrm{A}$ and $\mathrm{C}$ on the upper surface of the cylinder (i.e. at $y / D=0.5$ ) disappear concurrently and instead a saddle point $\mathrm{D}$ (Fig. 5d) is formed above the cylinder, i.e. at $y / D>0.5$ (Fig. 7b). An example of this change in the separation pattern is illustrated in Fig. 8 with a comparison between $R e=150$ (Fig. 8a) and $R e=160$ (Fig. 8b). The saddle point $\mathrm{D}$ is observed for all the cases beyond $R e=156$. 
(a)

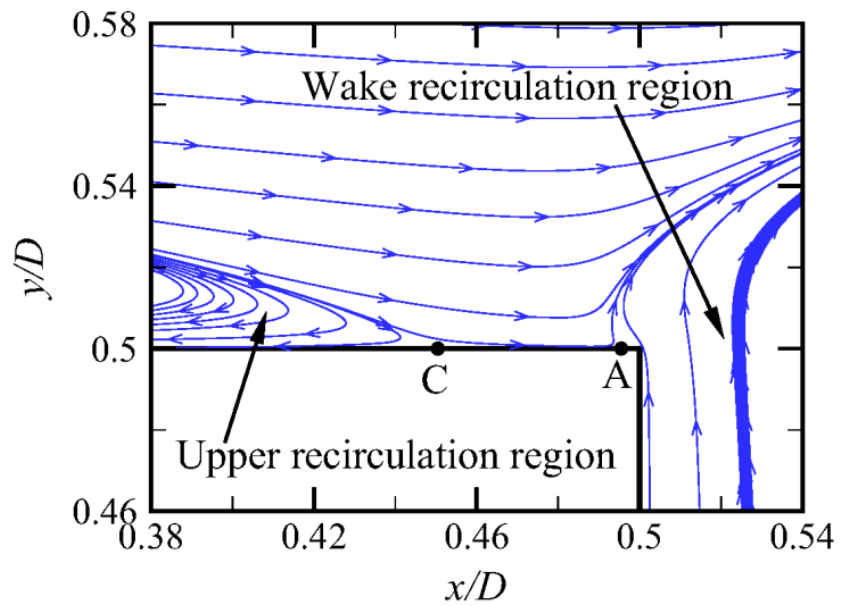

(b)

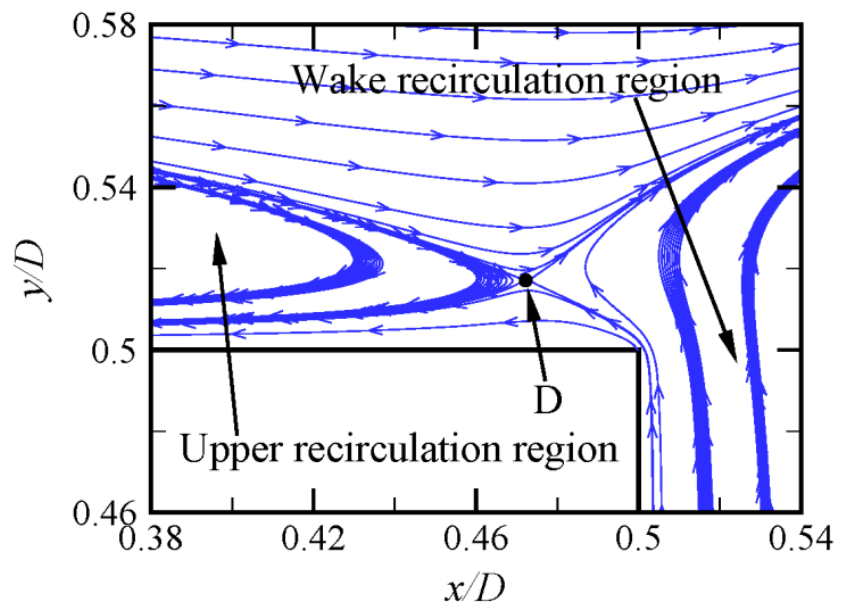

Fig. 8. Streamlines of the time-averaged flow for (a) $R e=150$, where the reattachment point $\mathrm{C}$ and separation point $\mathrm{A}$ are close to each other, and (b) $R e=160$, where points $\mathrm{C}$ and $\mathrm{A}$ disappear concurrently to give rise to the saddle point $\mathrm{D}$ above the cylinder. The streamlines are shown at near the upper right corner of the square cylinder.

With the emergence of the saddle point D away from the cylinder surface, a small amount of backward flow appears below the saddle point D (Fig. 5d,e; Fig. 8b). For $R e \geq 159$, Fig. 5(e) and Fig. 8(b) show that the backward flow originates from the flow entrainment from the wake recirculation region to the upper side of the cylinder. The two recirculation regions above the wake centreline, one in the upper half of the wake and one on the upper side of the cylinder, are further wrapped together by an outer recirculation region. For a small range of $R e=156-158$, the flow pattern shown in Fig. 5(d) is slightly different. Instead of showing flow entrainment from the 
wake recirculation region, the backward flow dives through the trailing edge slightly. This is because the streamlines have been calculated based on the time-averaged flow field. A further examination of the corresponding instantaneous flow fields shows that for certain phases the transient flow has flow entrainment from the wake recirculation region to the upper side of the cylinder, and the transient streamlines do not dive through the cylinder surface. Therefore, the flow patterns shown in Fig. 5(d) and Fig. 5(e) are deemed the same.

\section{Three-dimensional flows $(\operatorname{Re}>165.7)$}

Beyond the secondary wake instability at $R e=165.7$, the 3D flows are simulated with a reduced $F_{m r}$ of 1 so as to keep the computational cost in an affordable level. The corresponding 2D flows are simulated with both $F_{m r}=1$ and 4 so as to compare quantitatively with the $3 \mathrm{D}$ results and to quantify the effect of flow three-dimensionality.

As expected, the 2D results of the saddle point D calculated with $F_{m r}=1$ and 4 (Fig. 7b) show small differences due to the influence of mesh resolution. Nevertheless, both curves are smooth and show similar qualitative variation trends. To eliminate the small influence of mesh resolution, the following comparison between the $3 \mathrm{D}$ and $2 \mathrm{D}$ results is based on results both calculated with $F_{m r}=1$. As shown in Fig. 7(b), at the onset of the secondary wake instability, there is a sudden change in the location of the saddle point $\mathrm{D}$ ( of $\sim 0.033 \mathrm{D}$ ), owing to the subcritical nature of the mode A instability. For $R e=167-210$, although this sudden change in the location of the saddle point $\mathrm{D}$ results in a small difference between the $3 \mathrm{D}$ and $2 \mathrm{D}$ results of approximately $0.02-$ $0.03 \mathrm{D}$, the $3 \mathrm{D}$ and $2 \mathrm{D}$ results still follow similar variation trends (as indicated by a blue arrow in Fig. 7b). However, the 3D results deviate increasingly from their 2D counterparts for $R e=210-330$, which is similar to the increasing deviations between other 3D and 2D flow properties (e.g. the time-averaged drag coefficient, the root-mean-square lift coefficient and the wake formation length) observed in Jiang and Cheng (2018) and is due to the existence of two critical conditions at $R e \sim 210$ 
and 330 identified in Jiang and Cheng (2018).

As mentioned in section III.B.1, the emergence of the saddle point D is responsible for the backward flow beneath it. Figure 9 shows the 3D results of the horizontal and vertical components of the rate of flow ( $q_{x}$ and $\left.q_{y}\right)$ entrained from the wake recirculation region to the upper side of the cylinder. The flow rates are integrated over the vertical distance from the saddle point D (shown in Fig. 7b) down to the cylinder surface. It is found that the increase/decrease in the magnitude of $q_{x}$ corresponds to the upward/downward movement of the saddle point D shown in Fig. 7(b), while the increase/decrease in $q_{y}$ corresponds to the downstream/upstream movement of the saddle point $\mathrm{D}\left(q_{y}\right.$ is larger when the saddle point $\mathrm{D}$ is at more downstream locations because the streamlines near the trailing edge of the cylinder are more inclined). The saddle point D serves as a "valve" in controlling the rate of the entrained flow.

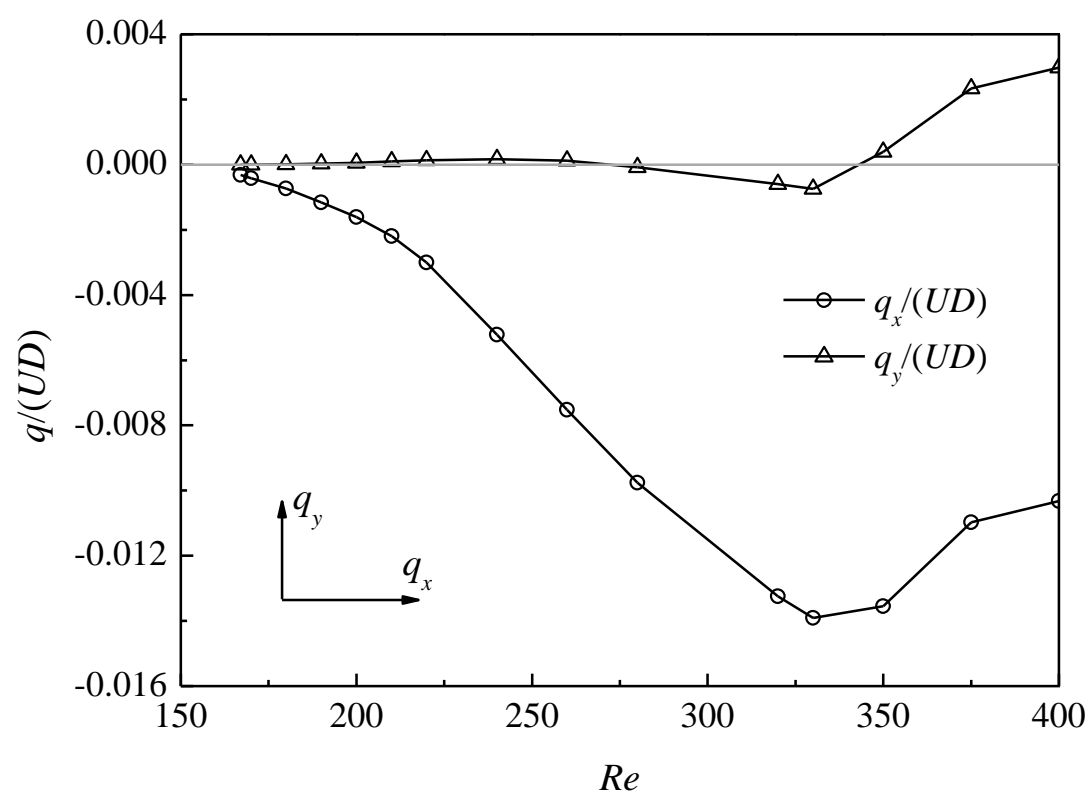

Fig. 9. Three-dimensional results of the horizontal and vertical components of the rate of flow ( $q_{x}$ and $q_{y}$ ) entrained from the wake recirculation region to the upper side of the cylinder.

Figure 10 compares the separation point $\mathrm{B}$ near the leading edge calculated through 3D (with $F_{m r}=1$ ) and 2D DNS (with both $F_{m r}=1$ and 4). Under the same $F_{m r}$ 
of 1 , the $3 \mathrm{D}$ and $2 \mathrm{D}$ simulations predict almost identical locations for the separation point B (see the inset of Fig. 10), which suggests that the separation point B is almost unaffected by the effect of flow three-dimensionality. Therefore, it is anticipated that the separation point B predicted by 2D DNS with $F_{m r}=4$ shown in Fig. 7(a) and Fig. 10 represents also the 3D results with the same resolution of $F_{m r}=4$.

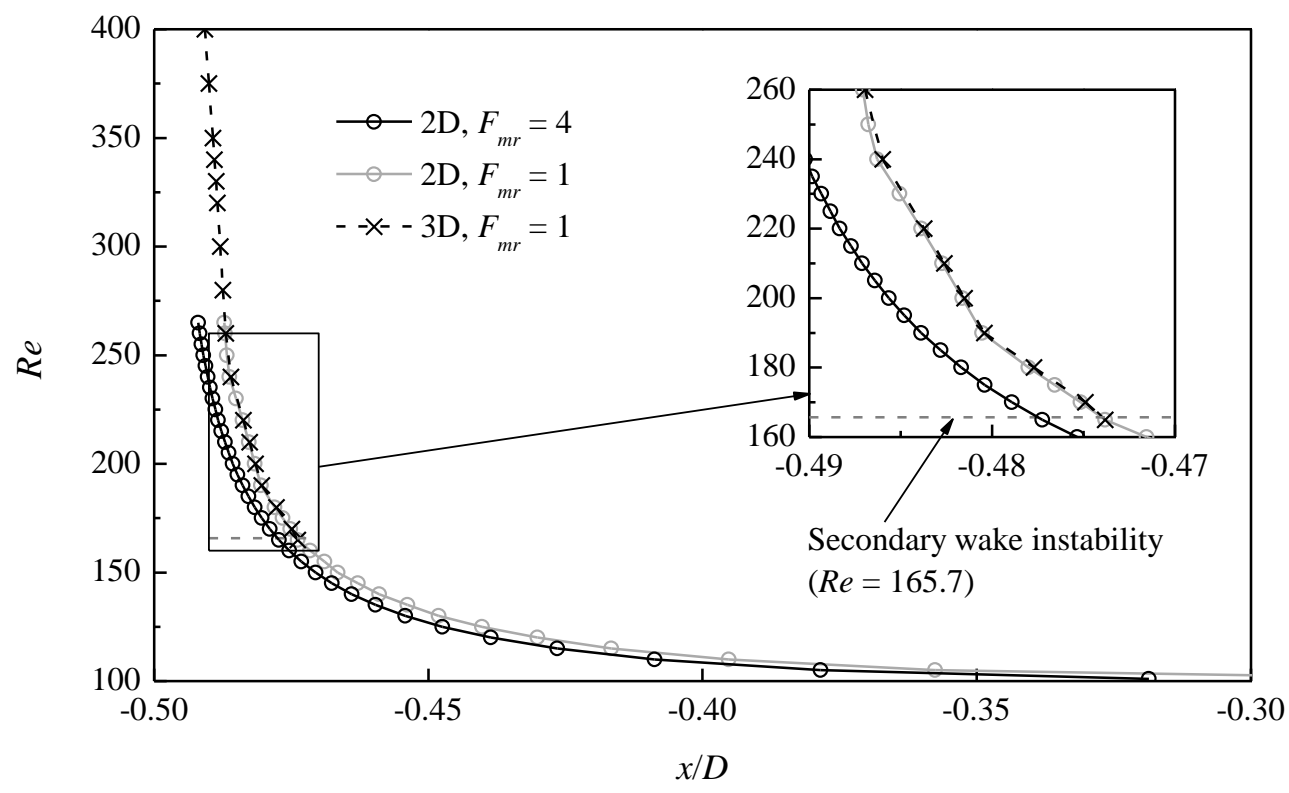

Fig. 10. Movement of the time-averaged separation point $\mathrm{B}$ with the evolution of $R e$.

It is interesting to note that the flow three-dimensionality at $R e>165.7$ affects the location of the saddle point D (Fig. 7b) but has almost no influence on the location of the separation point B (Fig. 10). Physically, the flow three-dimensionality is induced by the development of 3D flow structures in the near wake of the cylinder. Figure 11 shows instantaneous streamwise and spanwise vorticity fields developed in the near wake of a square cylinder for $R e=170$, where the streamwise vorticity $\left(\omega_{x}\right)$ and spanwise vorticity $\left(\omega_{z}\right)$ are defined as

$$
\begin{aligned}
& \omega_{x}=\left(\frac{\partial u_{z}}{\partial y}-\frac{\partial u_{y}}{\partial z}\right) \frac{D}{U}, \\
& \omega_{z}=\left(\frac{\partial u_{y}}{\partial x}-\frac{\partial u_{x}}{\partial y}\right) \frac{D}{U} .
\end{aligned}
$$

The iso-surfaces of the streamwise vorticity shown in Fig. 11(a,b) reveal the 3D flow 
structure of mode A with vortex dislocations. According to Williamson (1996), Leweke and Williamson (1998) and Thompson et al. (2001), mode A originates in the primary vortex cores and induces streamwise vortices in the braid shear layers between the primary vortex cores, while mode B originates in the braid shear layers and generates streamwise vortices in there. In other words, the modes A and B structures would only develop after the formation of the primary vortex cores. Figure 11(c) shows the spanwise vorticity field of the span-averaged flow, which highlights the locations of the primary vortex cores. The dashed lines in Fig. 11(c) indicate the locations for the streamwise vortices shown in Fig. 11(a,b), which appear between the primary vortex cores. The latest-generated primary vortex core shown in Fig. 11(c) is a negatively-signed one just rolled up from the upper separating shear layer (at $x / D \sim$ $1-3$ ), where no braid shear layer exists upstream of it for the development of the mode A/B structure. It is also seen in Fig. 11(a,b) that the streamwise vortices generally emerge at $x / D \gtrsim 1.5$. 
(a)

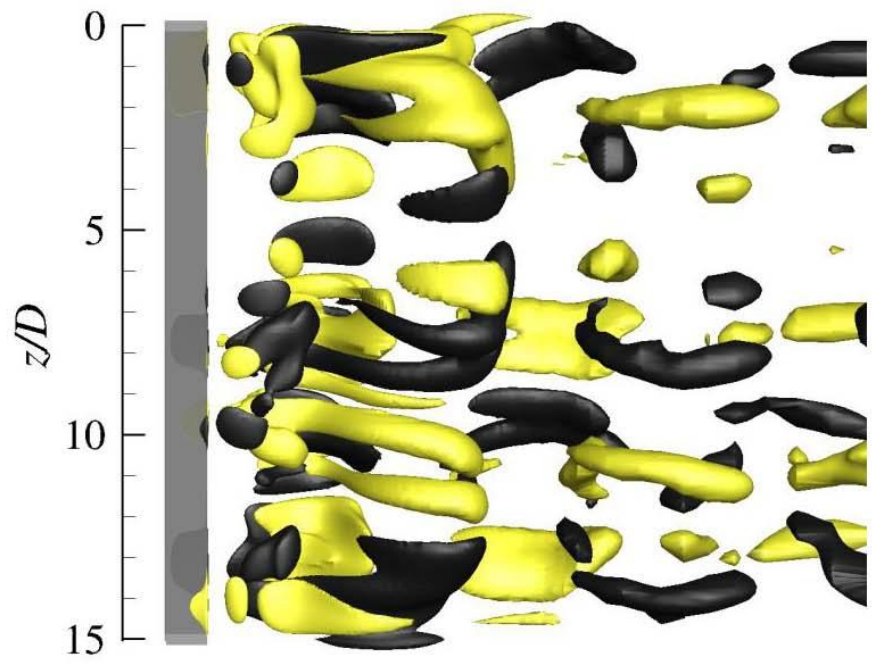

(b)

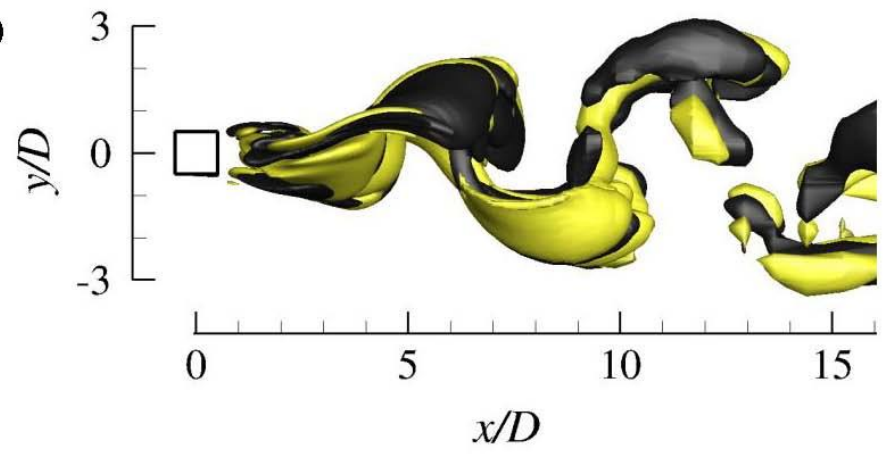

(c)

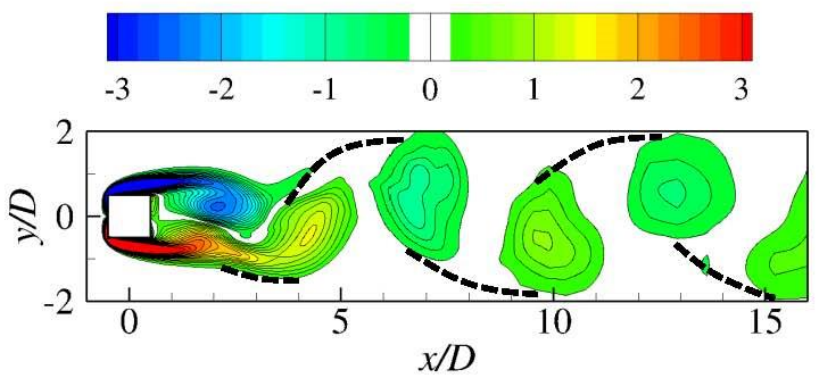

Fig. 11. Instantaneous vorticity fields in the near wake of a square cylinder for $R e=$ 170. Panels (a) and (b) show iso-surfaces of the streamwise vorticity with $\omega_{x}=+0.4$ (dark grey) and -0.4 (light yellow), while panel (c) shows spanwise vorticity field of the span-averaged flow.

The development of the $3 \mathrm{D}$ structures at $x / D \gtrsim 1.5$ affects the degree of three-dimensionality of the ambient flow (including the upstream flow), which is quantified through the contours of $u_{z}{ }^{\prime} u_{z}{ }^{\prime} / U^{2}$ of the span-averaged 3D flow (Fig. 12a), where $u_{z}^{\prime}$ is the root-mean-square of the spanwise velocity defined as 
$u_{z}^{\prime}=\sqrt{\frac{1}{N} \sum_{i=1}^{N}\left(u_{z, i}-\overline{u_{z}}\right)^{2}}$,

where $N$ is the number of time steps used for the statistics, and $\overline{u_{z}}$ is the time-averaged spanwise velocity (which is equal to zero). As shown in Fig. 12(a), for $R e=170$ the strongest fluctuation of $u_{z}$ occurs at $x / D \sim 2.3-3.5$. With distance away from this area, the fluctuation of $u_{z}$ reduces. The $u_{z}^{\prime} u_{z}^{\prime} / U^{2}$ values near the locations of the saddle point $\mathrm{D}$ and separation point $\mathrm{B}$ are in the orders of $10^{-4}$ and $10^{-6}$, respectively. The $u_{z}^{\prime} u_{z}^{\prime} / U^{2}$ values of $10^{-4}$ and $10^{-6}$ are equivalent to sinusoidal fluctuations of $u_{z}$ with amplitudes of $\pm 1.41 \times 10^{-2} U$ and $\pm 1.41 \times 10^{-3} U$, respectively. The separation point $\mathrm{B}$ is unaffected by the flow three-dimensionality developing at $R e>$ 165.7 because the 3D flow structures developed in the wake region has little influence on the flow three-dimensionality back to the leading edge of the cylinder. In contrast, at the location of the saddle point $\mathrm{D}$, the $u_{z}^{\prime} u_{z}^{\prime} / U^{2}$ value increases by approximately two orders of magnitude, such that there is a small difference in the location of the saddle point $\mathrm{D}(\sim 0.03 \mathrm{D})$ between the $2 \mathrm{D}$ and $3 \mathrm{D}$ results shown in Fig. 7(b). The above conclusions are applicable up to the largest $R e$ of 400 considered in the present study. As shown in Fig. 12(a,b), the $u_{z}^{\prime} u^{\prime} / U^{2}$ fields of $R e=170$ and 400 display similar patterns.

(a)

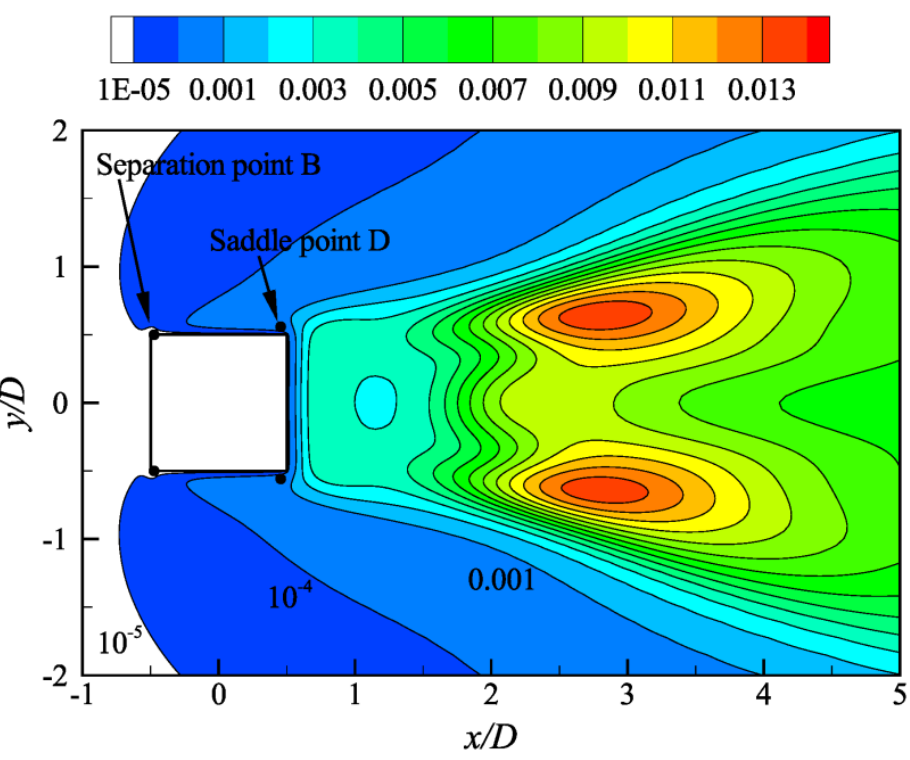


(b)

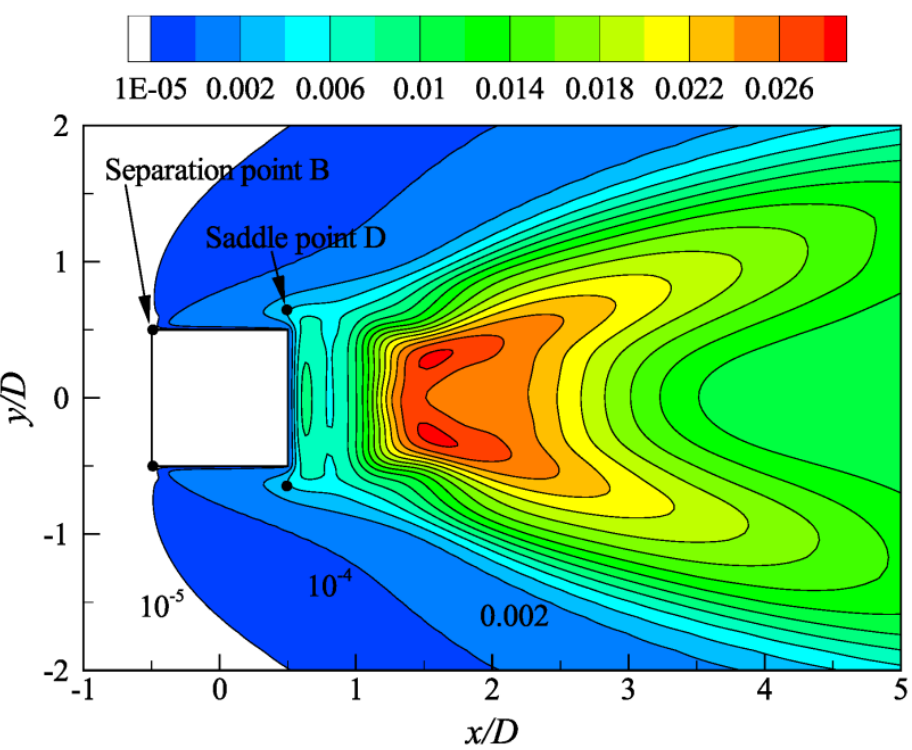

(c)

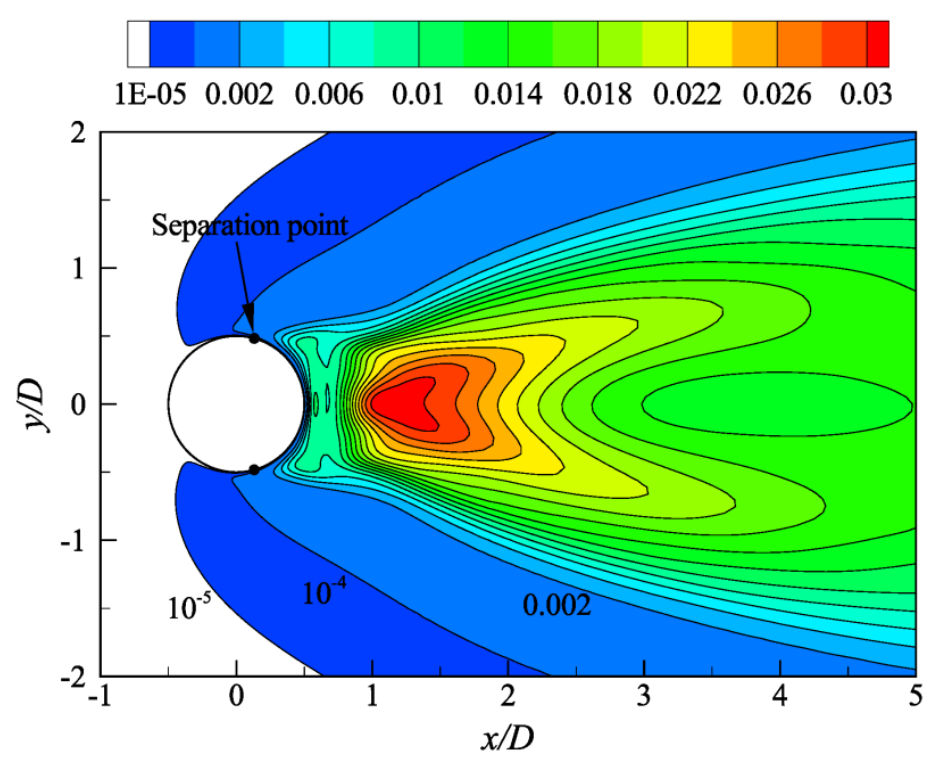

Fig. 12. Contours of $u_{z}^{\prime} u_{z}^{\prime} / U^{2}$ of the span-averaged 3D flow for (a) flow past a square cylinder at $R e=170$, (b) flow past a square cylinder at $R e=400$, and (c) flow past a circular cylinder at $R e=400$.

In contrast, Jiang (2020) showed that for the case of a circular cylinder the flow three-dimensionality occurring at $R e \gtrsim 190$ does affect the location of the separation point. For example, at $R e=400$ the difference in the separation angle between the $3 \mathrm{D}$ and $2 \mathrm{D}$ results is $3.6^{\circ}$. To examine the difference between a square and a circular cylinder, Fig. 12(b,c) compares the $u_{z}^{\prime} u_{z}^{\prime} / U^{2}$ fields for both cylinders at $R e=400$. In contrast to the separation near the leading edge for a square cylinder, the separation for a circular cylinder occurs more downstream, such that it is more affected by the 
flow three-dimensionality induced by the 3D flow structures developed in the wake region. This is evidenced by a comparison of the $u_{z}^{\prime} u_{z}^{\prime} / U^{2}$ values near the separation points for a circular and a square cylinder, where the former is approximately two orders of magnitude larger than the latter.

\section{Instantaneous separation and reattachment locations}

\section{Two-dimensional flows $(\operatorname{Re}<165.7)$}

Fig. 13 shows the time evolution of the instantaneous separation and reattachment locations on the upper surface of the square cylinder over a vortex shedding period $T$. The open and filled symbols in Fig. 13 represent the separation and reattachment locations, respectively. The time evolution of the instantaneous separation and reattachment locations on the lower surface of the cylinder has a $0.5 T$ phase difference from those on the upper surface and is omitted for simplicity.

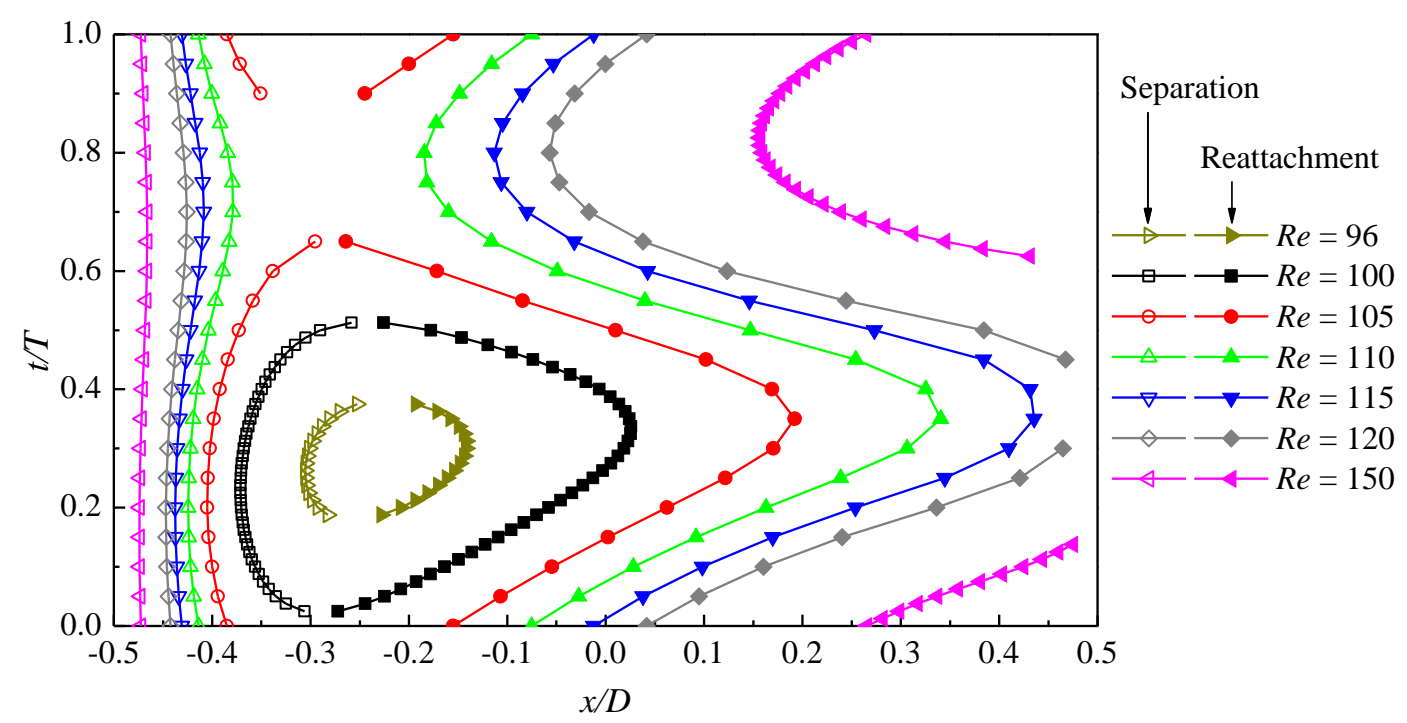

Fig. 13. Time evolution of the instantaneous separation and reattachment locations on the upper surface of the square cylinder over a vortex shedding period.

As shown in Fig. 13, the separation near the leading edge and the recirculation region on the upper surface of the cylinder are first detected at $R e=96$ (but not at $R e$ $=95$ ). For $R e=96-107$, this recirculation region exists for only some of the phases 
in a vortex shedding period, over which it emerges, expands, shrinks and disappears. For $R e=108-118$, this recirculation region exists throughout. For $R e \geq 119$, due to the further expansion of the recirculation region towards the trailing edge of the cylinder for some of the phases, the reattachment point on the cylinder surface may sometimes disappear and be replaced by a saddle point away from the cylinder surface. The emergence of the saddle point results in the wake flow entrainment for the corresponding phases. Table 3 summarises the critical Re values for the onset of some flow patterns for the time-averaged and instantaneous flows. The critical Re values for the instantaneous flows are smaller than those for the time-averaged flows, since for the instantaneous flows the flow patterns may appear for only some of the phases.

Table 3. Critical $R e$ values for the onset of some flow patterns for the time-averaged and instantaneous flows.

\begin{tabular}{lll}
\hline Flow pattern & $\begin{array}{l}\text { Time-averaged } \\
\text { flow }\end{array}$ & $\begin{array}{l}\text { Instantaneous } \\
\text { flow }\end{array}$ \\
\hline $\begin{array}{l}\text { Onset of the separation point B, the reattachment } \\
\text { point C and the recirculation region on the }\end{array}$ & 100.36 & 96 \\
$\begin{array}{l}\text { upper/lower surface of the cylinder } \\
\text { Onset of the saddle point D and wake flow }\end{array}$ & 156 & 119 \\
entrainment & & \\
\hline
\end{tabular}

Figure 14 shows the time evolution of the streamline pattern around a square cylinder for $R e=110$ (Fig. 14a-e) and $R e=150$ (Fig. 14f-j). In accordance with the alternate vortex shedding process, two wake recirculation regions (denoted as 1 and 2 in Fig. 14) develop alternately from the two trailing edges of the cylinder over a time period of $T$. This phenomenon is similar to the case of a circular cylinder, as shown in Fig. 15 with an example at $R e=150$. However, a major difference between a square and a circular cylinder is that for a square cylinder two additional recirculation 
regions develop on the upper and lower surfaces of the cylinder at $R e \geq 96$, owing to the geometric sharp corners of the square cross-section (see also comparisons between circular and square cylinders in Zafar and Alam (2019) and Alam et al. (2020)). For $R e=96-118$ (e.g. Fig. 14a-e), the two additional recirculation regions expand and shrink cyclically on the upper and lower surfaces of the cylinder (as quantified in Fig. 13), without interaction with the two wake recirculation regions. As Re exceeds 119 (e.g. Fig. 14f-j), the further expanded upper and lower recirculation regions may sometimes merge with the wake recirculation regions 2 and 1, respectively (e.g. Fig. 14f,i). In addition, flow entrainment from the wake to the upper/lower side of the cylinder is observed (e.g. Fig. 14g,h,j). 
(a)

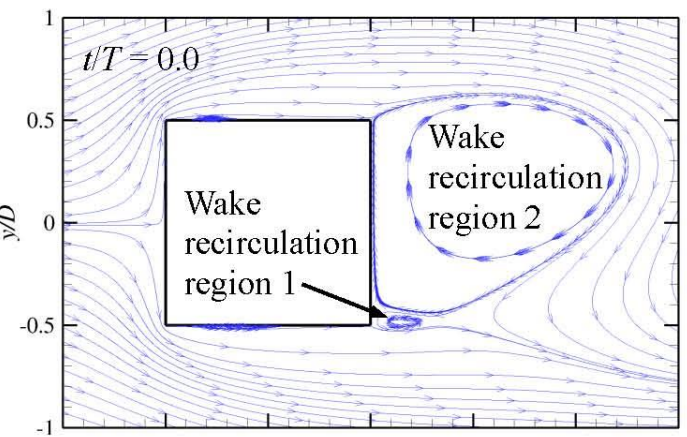

(b)

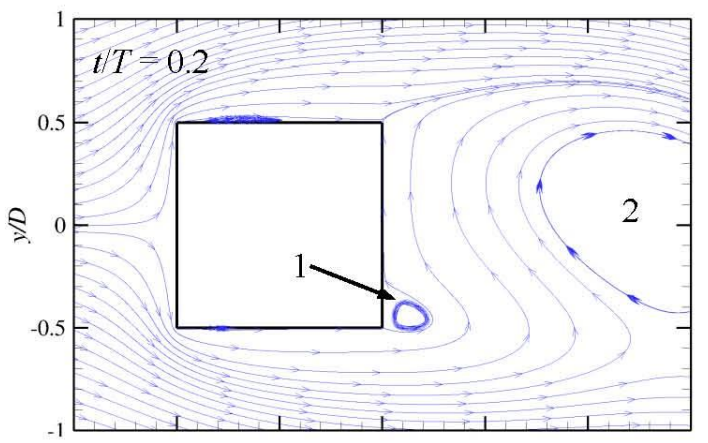

(c)

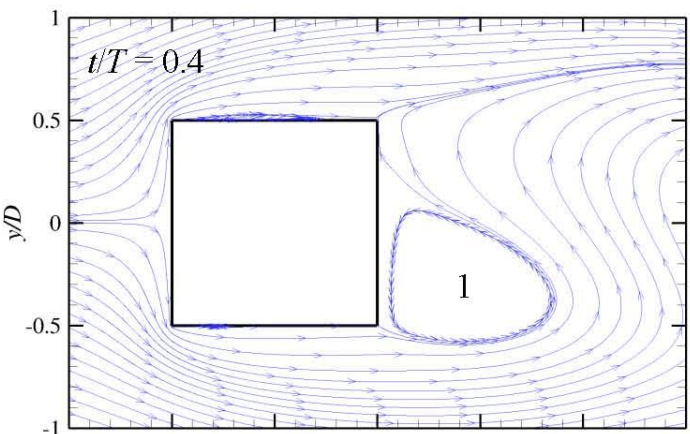

(d)

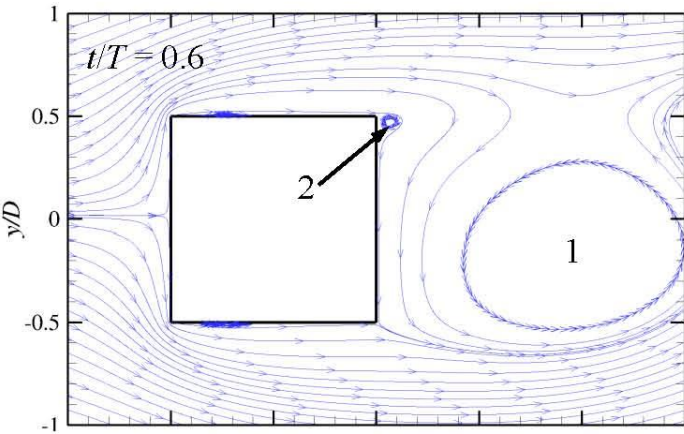

(e)

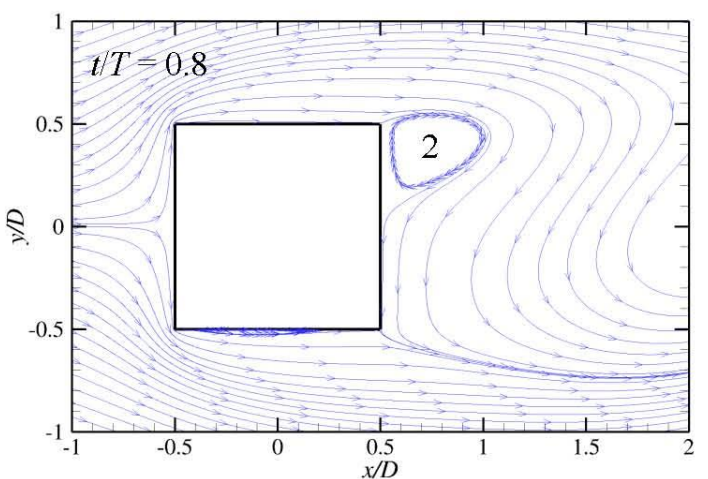

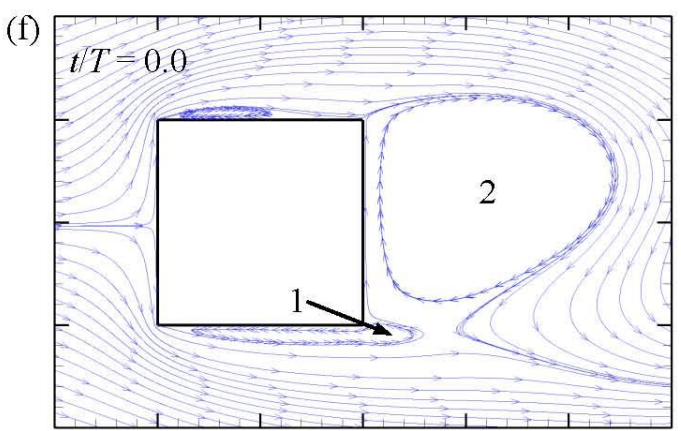

(g)

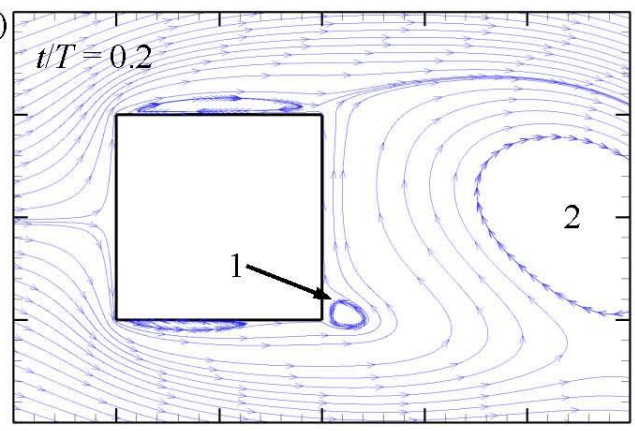

(h)

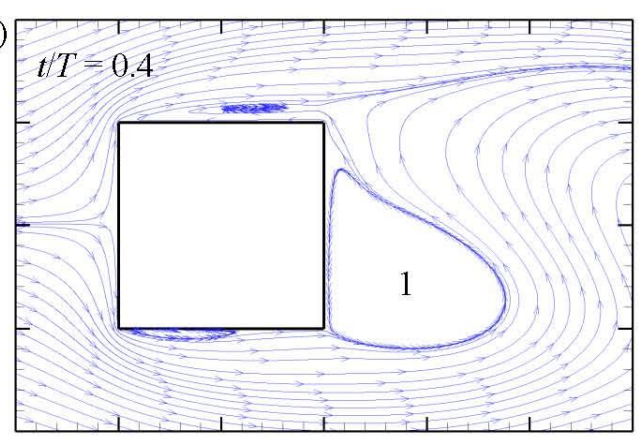

(i)

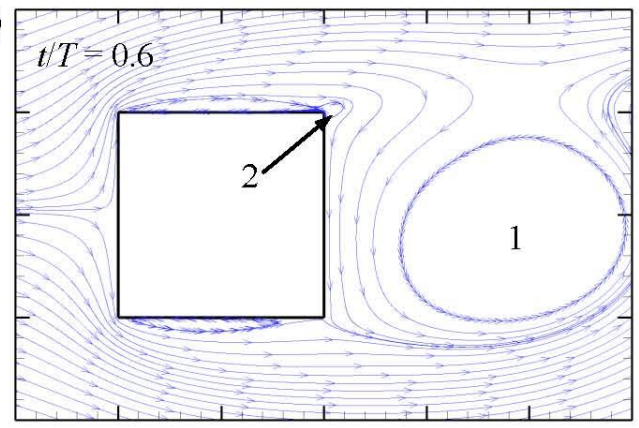

(j)

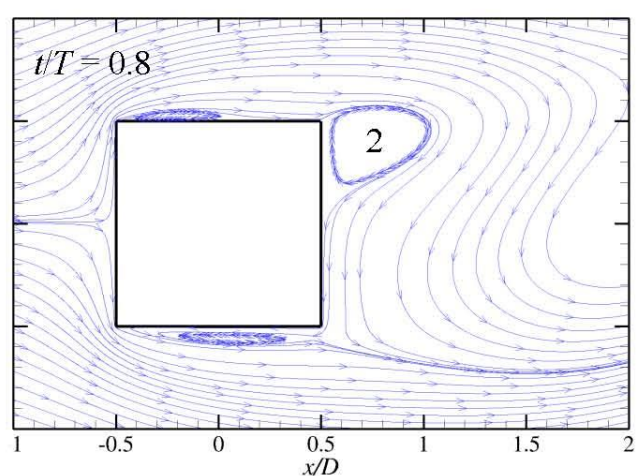

Fig. 14. Time evolution of the streamline pattern around a square cylinder over a vortex shedding period. (a) - (e) for $R e=110$, and (f) $-(\mathrm{j})$ for $R e=150$. 
(a)

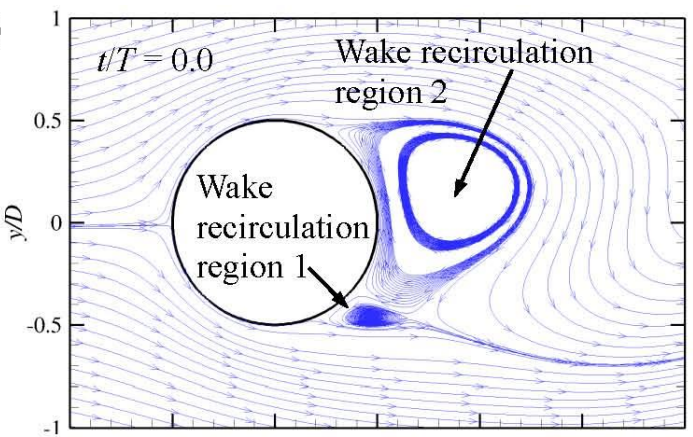

(b)

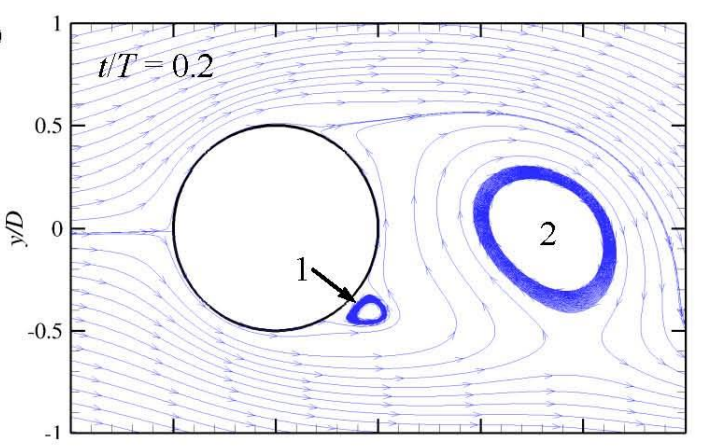

(c)

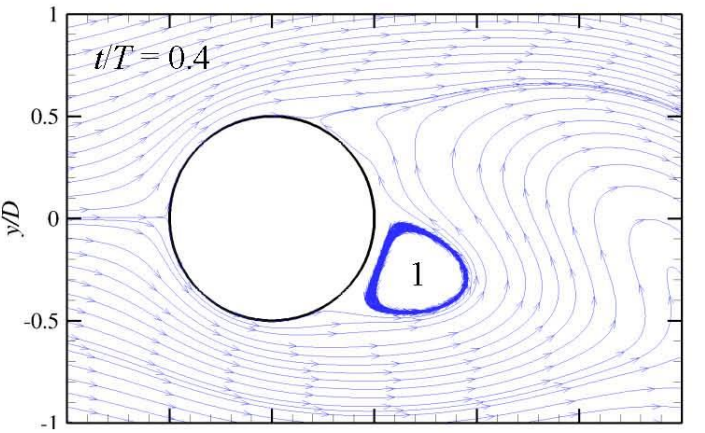

(d)

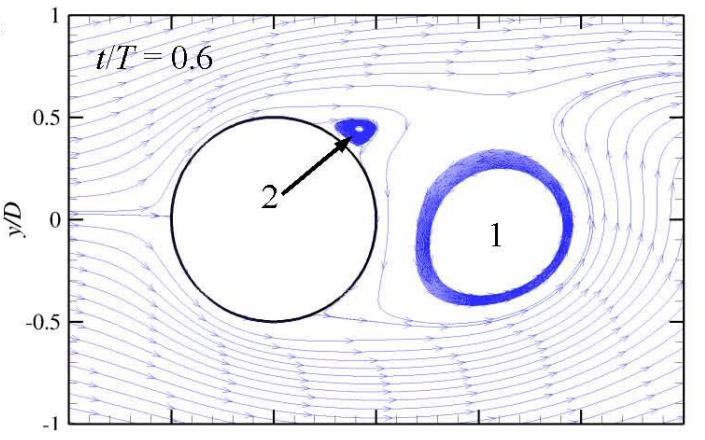

(e)

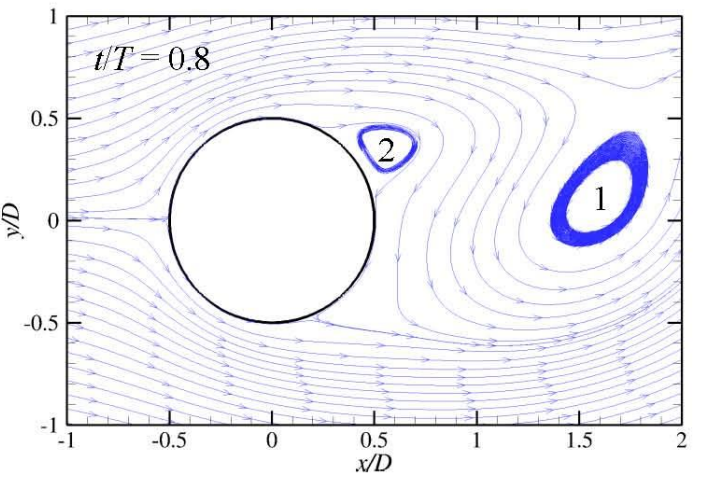

Fig. 15. Time evolution of the streamline pattern around a circular cylinder over a vortex shedding period (from panels (a) to (e) with an interval of $0.2 T$ ) for $R e=150$. 


\section{Three-dimensional flows $(\operatorname{Re}>165.7)$}

The instantaneous separation locations beyond the secondary wake instability of $R e=165.7$ is examined with an example at $R e=200$. Figure 16(a) shows the time variation of the span-averaged upper and lower separation locations for $R e=200$ over 31 vortex shedding cycles. A comparison with the corresponding variation range calculated through 2D DNS (marked by the horizontal dashed lines in Fig. 16(a)) indicates that the flow three-dimensionality would induce a very slight $(\sim 0.0013 D)$ reduction in the variation range of the separation location.
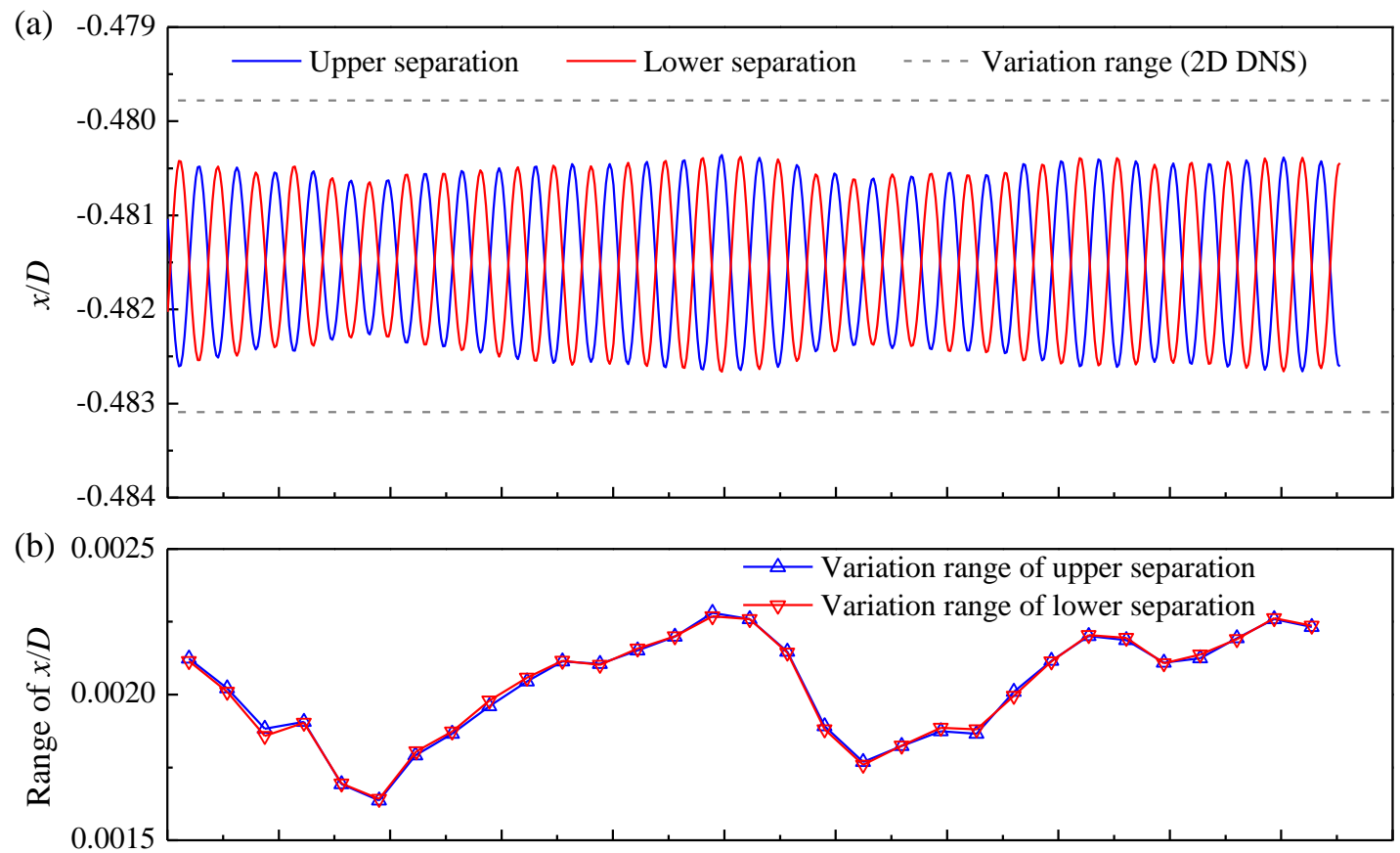

(c)

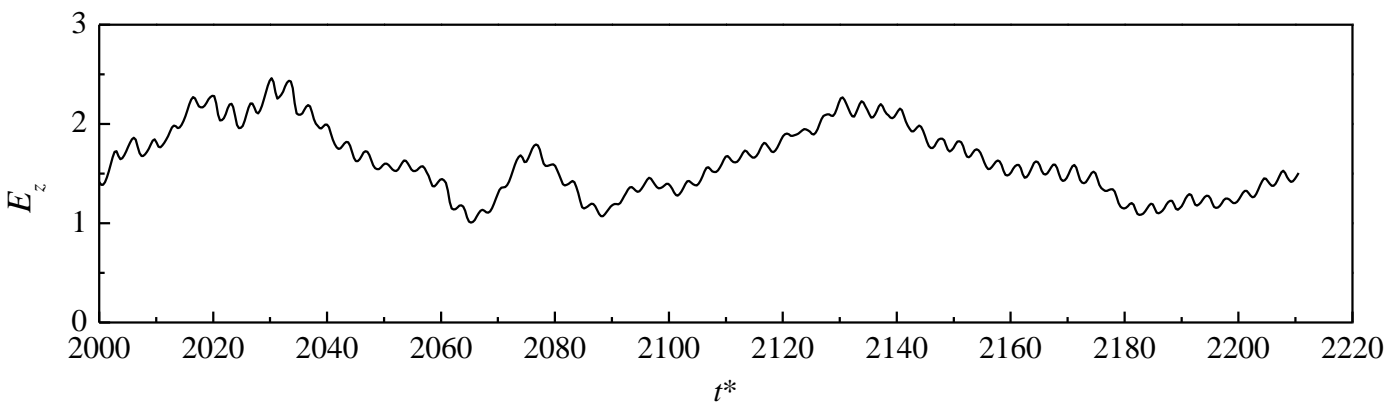

Fig. 16. An example case of flow past a square cylinder at $R e=200$. (a) Time variation of the span-averaged upper and lower separation locations, together with the corresponding variation range calculated through 2D DNS (with $F_{m r}=1$ ) marked by 
the horizontal dashed lines, (b) time variation of the 3D variation ranges of the span-averaged upper and lower separation locations, and (c) time variation of the spanwise energy in the near wake.

The 3D variation range is further quantified in Fig. 16(b), while the flow three-dimensionality is quantified in Fig. $16(\mathrm{c})$ by the spanwise energy $E_{z}$ defined as:

$E_{z}=\frac{1}{2} \int_{V}\left(u_{z} / U\right)^{2} \mathrm{~d} V$

where $V$ is the volume of the flow field of interest, which adopts the near-wake region of $x / D=0-10$ for the development of the 3D wake structures. In general, larger flow three-dimensionality in the near wake would result in smaller variation range of the separation location.

Figure 17 shows the variation range of the instantaneous upper/lower separation location for different $R e$ values, calculated through 2D DNS with $F_{m r}=4$. The variation range reduces with increase in $R e$. For the $3 \mathrm{D}$ flows at $R e>165.7$, the time-averaged separation location is well represented by the 2D result (see Fig. 10), while the example at $R e=200$ shown in Fig. 16 suggests that the 3D variation range is slightly smaller than its counterpart calculated in 2D, i.e. the shaded area in Fig. 17 would reduce slightly. The exact amount of reduction is not quantified by 3D DNS with $F_{m r}=4$, since the computational cost would be impractically expensive. The results shown in Fig. 17 suggest that with increase in $R e$ the separation location (both time-averaged and instantaneous) converges towards the leading edge of the cylinder. For example, at $R e=240$ the time-averaged separation location is only $0.01 D$ away from the leading edge while the variation range is smaller than $0.005 D$, such that in practice the separation location may be indistinguishable from the leading edge. 


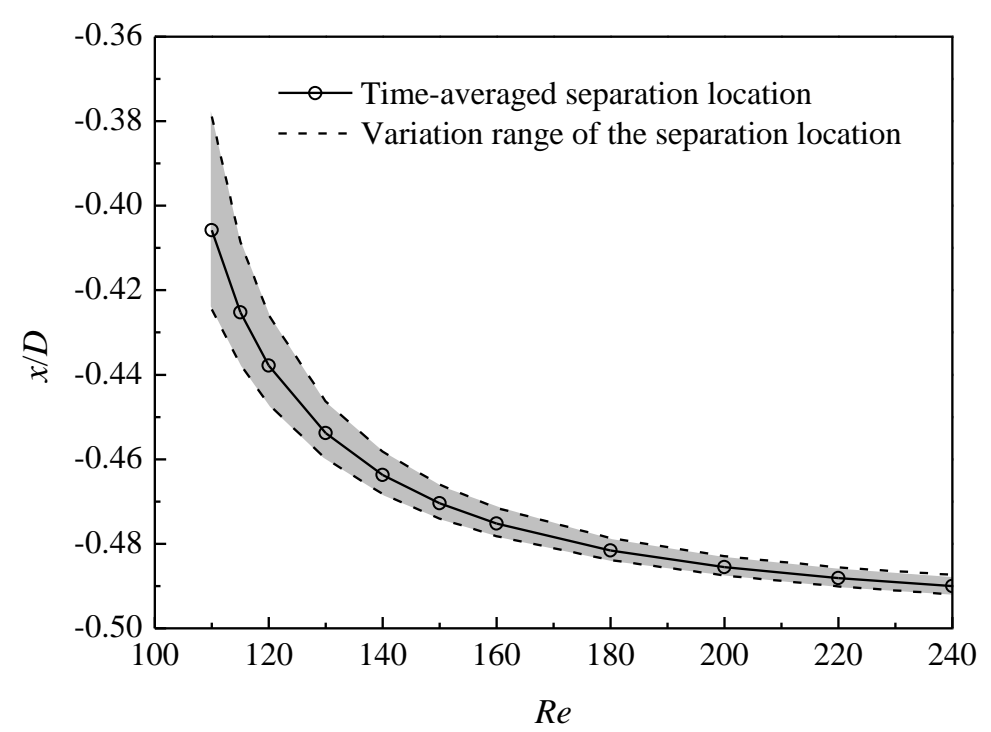

Fig. 17. Variation range of the instantaneous upper/lower separation location for different $R e$ values. The variation range is shaded in grey. The results are calculated through 2D DNS with $F_{m r}=4$ but may well represent the 3D results.

The gradual movement of the separation location from the cylinder surface to the corner with increasing $R e$ is a common phenomenon that has been observed for some other sharp-cornered bluff bodies, e.g. an inclined square cylinder (Yoon, et al., 2010) and an inclined triangular cylinder ( $\mathrm{Ng}$ et al., 2016). We believe that the sharp corner of a bluff body is where the separation location can hardly climb over and therefore converges to, but may not be the location for the initiation of separation. In the present study, this phenomenon has been generalised to the case of a square cylinder.

\section{Conclusions}

This paper investigates the flow (of a Newtonian and incompressible fluid) separation around a square cylinder for $R e=10-400$ through DNS. The evolution of the separation pattern with $R e$ is categorised with a fine $R e$ resolution of 1 . In particular, both time-averaged and instantaneous separation patterns show that the flow may not separate at the sharp corners for a certain range of moderate $R e$ values. Instead, the separation location gradually converges towards the leading edge with increasing $R e$, as may be a common phenomenon for sharp-cornered bluff bodies. 
Based on the time-averaged flows, it is found that the flow separation near the leading edge first appears at $R e=100.36$ at $x / D=-0.271$. With the increase in $R e$ from 100.36 to 155 , this separation point moves upstream while the corresponding reattachment point moves downstream, which indicates a gradual increase in the size of the recirculation region on the upper/lower surface of the cylinder. The increase in the size of the recirculation region eventually results in the concurrent disappearance of the reattachment point and rear separation point on the upper/lower surface and instead the emergence of a saddle point away from the cylinder surface for $R e \geq 156$. The emergence of the saddle point results in a small amount of backward flow on the upper/lower side of the cylinder, owing to the flow entrainment from the wake recirculation region. The rate of the entrained flow is controlled by the location of the saddle point.

For the instantaneous flows, the critical $R e$ values for the onset of (i) the flow separation near the leading edge and (ii) the saddle point and wake flow entrainment are decreased from 100.36 and 156 to 96 and 119, respectively, since for the instantaneous flows these flow patterns may appear for only some of the phases. With the increase in $R e$, the instantaneous variation range of the separation location near the leading edge reduces.

It is found that the flow three-dimensionality developing at $R e>165.7$ affects the location of the saddle point but has almost no influence on the location of the separation point. This is because the $3 \mathrm{D}$ flow structures developed in the wake region has little influence on the flow three-dimensionality back to the separation location near the leading edge. In contrast, for a circular cylinder the separation location is noticeably affected by the flow three-dimensionality, since the separation for a circular cylinder occurs more downstream than that of a square cylinder.

\section{Data Availability Statement}

Raw data were generated at the Pawsey Supercomputing Centre. Derived data supporting the findings of this study are available from the corresponding author upon 
reasonable request.

\section{Acknowledgements}

The first author would like to acknowledge the support from the Australian Research Council through the DECRA scheme (Grant No. DE190100870). This work was supported by resources provided by the Pawsey Supercomputing Centre with funding from the Australian Government and the Government of Western Australia.

\section{References}

Achenbach, E., "Distribution of local pressure and skin friction around a circular cylinder in cross-flow up to $R e=5 \times 10^{6}$," Journal of Fluid Mechanics 34, 625-639 (1968).

Alam, M.M., Abdelhamid, T., Sohankar, A., "Effect of cylinder corner radius and attack angle on heat transfer and flow topology," International Journal of Mechanical Sciences 175, 105566 (2020).

Bai, H., Alam, M.M., "Dependence of square cylinder wake on Reynolds number," Physics of Fluids 30, 015102 (2018).

Barkley, D., Henderson, R.D., “Three-dimensional Floquet stability analysis of the wake of a circular cylinder," Journal of Fluid Mechanics 322, 215-241 (1996).

Cantwell, C.D., et al., "Nektar++: An open-source spectral/hp element framework," Computer Physics Communications 192, 205-219 (2015).

Choi, C., Jang, Y., Yang, K., "Secondary instability in the near-wake past two tandem square cylinders," Physics of Fluids 24, 024102 (2012).

Jiang, H., "Separation angle for flow past a circular cylinder in the subcritical regime," Physics of Fluids 32, 014106 (2020).

Jiang, H., Cheng, L., "Hydrodynamic characteristics of flow past a square cylinder at moderate Reynolds numbers,” Physics of Fluids 30, 104107 (2018).

Jiang, H., Cheng, L.. "Transition to the secondary vortex street in the wake of a circular cylinder," Journal of Fluid Mechanics 867, 691-722 (2019).

Jiang, H., Cheng, L., An, H., “Three-dimensional wake transition of a square cylinder,” Journal of 
Fluid Mechanics 842, 102-127 (2018).

Jiang, H., Cheng, L., Draper, S., An, H., "Prediction of the secondary wake instability of a circular cylinder with direct numerical simulation," Computers and Fluids 149, 172-180 (2017).

Jiang, H., Cheng, L., Draper, S., An, H., Tong, F., “Three-dimensional direct numerical simulation of wake transitions of a circular cylinder," Journal of Fluid Mechanics 801, 353-391 (2016).

Kumar, D., Sourav, K., Sen, S., Yadav, P.K., "Steady separation of flow from an inclined square cylinder with sharp and rounded base," Computers and Fluids 171, 29-40 (2018).

Leweke, T., Williamson, C.H.K., “Three-dimensional instabilities in wake transition,” European Journal of Mechanics - B/Fluids 17, 571-586 (1998).

Ng, Z.Y., Vo, T., Hussam, W.K., Sheard, G.J., "Two-dimensional wake dynamics behind cylinders with triangular cross-section under incidence angle variation," Journal of Fluids and Structures 63, 302-324 (2016).

Ozgoren, M., "Flow structure in the downstream of square and circular cylinders," Flow Measurement and Instrumentation 17, 225-235 (2006).

Park, D., Yang, K., "Flow instabilities in the wake of a rounded square cylinder," Journal of Fluid Mechanics 793, 915-932 (2016).

Posdziech, O., Grundmann, R., "Numerical simulation of the flow around an infinitely long circular cylinder in the transition regime," Theoretical and Computational Fluid Dynamics 15, $121-141(2001)$.

Robichaux, J., Balachandar, S., Vanka, S.P., “Three-dimensional Floquet instability of the wake of square cylinder," Physics of Fluids 11, 560-578 (1999).

Saha, A.K., Muralidhar, K., Biswas, G., "Transition and chaos in two-dimensional flow past a square cylinder,” Journal of Engineering Mechanics 126, 523-532 (2000).

Sen, S., Mittal. S., Biswas, G., "Steady separated flow past a circular cylinder at low Reynolds numbers," Journal of Fluid Mechanics 620, 89-119 (2009).

Sen, S., Mittal. S., Biswas, G., "Flow past a square cylinder at low Reynolds numbers," International Journal for Numerical Methods in Fluids 67, 1160-1174 (2011).

Sharma, A., Eswaran, V., "Heat and fluid flow across a square cylinder in the two-dimensional laminar flow regime,” Numerical Heat Transfer, Part A: Applications 45, 247-269 (2004).

Sheard, G.J., Fitzgerald, M.J., Ryan, K., "Cylinders with square cross-section: wake instabilities 
with incidence angle variation,” Journal of Fluid Mechanics 630, 43-69 (2009).

Sohankar, A., Norberg, C., Davidson, L., "Numerical simulation of unsteady low-Reynolds number flow around rectangular cylinders at incidence," Journal of Wind Engineering and Industrial Aerodynamics 69-71, 189-201 (1997).

Sohankar, A., Norberg, C., Davidson, L., "Simulation of three-dimensional flow around a square cylinder at moderate Reynolds numbers," Physics of Fluids 11, 288-306 (1999).

Thompson, M.C., Leweke, T., Williamson, C.H.K., "The physical mechanism of transition in bluff body wakes," Journal of Fluids and Structures 15, 607-616 (2001).

Wieselsberger, C., "New data on the laws of fluid resistance," NACA Technical Note 84. National Advisory Committee for Aeronautics, Washington, DC, United States (1922).

Williamson, C.H.K., "Vortex dynamics in the cylinder wake," Annual Review of Fluid Mechanics 28, 477-539 (1996).

Wu, M.H., Wen, C.Y., Yen, R.H., Weng, M.C., Wang, A.B., "Experimental and numerical study of the separation angle for flow around a circular cylinder at low Reynolds number," Journal of Fluid Mechanics 515, 233-260 (2004).

Yoon, D.H., Yang, K.S., Choi, C.B., "Flow past a square cylinder with an angle of incidence," Physics of Fluids 22, 043603 (2010).

Zafar, F., Alam, M.M., "Flow structure around and heat transfer from cylinders modified from square to circular," Physics of Fluids 31, 083604 (2019). 